\title{
Global Stability, Limit Cycles and Chaotic Behaviors of Second Order Interpolative Sigma Delta Modulators
}

\author{
Charlotte Yuk-Fan Ho \\ Telephone: +44 (0)20 78825555 ext. 4333 Fax: +44 (0)20 78827997 Email: c.ho@qmul.ac.uk \\ School of Mathematical Sciences, Queen Mary, University of London, Mile End Road, London, E1 4NS, \\ United Kingdom. \\ *Bingo Wing-Kuen Ling \\ Telephone: +44 (0)1522668901 Fax: +44 (0)1522886489 Email: wling@post01.lincoln.ac.uk \\ School of Engineering, University of Lincoln, Lincoln, Lincolnshire, LN6 7TS, United Kingdom. \\ Joshua D. Reiss \\ Telephone:+44(0)2078827982 Fax:+44(0)2078827997 Email: josh.reiss@elec.qmul.ac.uk \\ Department of Electronic Engineering, Queen Mary, University of London, Mile End Road, London, E1 \\ $4 N S$, United Kingdom. \\ Xinghuo $\mathrm{Yu}$ \\ Telephone +61 (0)399255317 Fax: +61 (0)399255310 Email: x.yu@ rmit.edu.au
}

School of Electrical and Computer Engineering, Royal Melbourne Institute of Technology, GPO Box 2476V, Melbourne, VIC 3001, Australia.

\begin{abstract}
It is well known that second order lowpass interpolative sigma delta modulators (SDMs) may suffer from instability and limit cycle problems when the magnitudes of the input signals are at large and at intermediate levels, respectively. In order to solve these problems, we propose to replace the second order lowpass interpolative SDMs to a specific class of second order bandpass interpolative SDMs with the natural frequencies of the loop filters very close to zero. The global stability property of this class of second order bandpass interpolative SDMs is characterized and some interesting phenomena are discussed. Besides, conditions for the occurrence of limit cycle and fractal behaviors are also derived, so that these unwanted behaviors will not happen or can be avoided. Moreover, it is found that these bandpass SDMs may exhibit irregular and conical-like chaotic patterns on the phase plane. By utilizing these chaotic behaviors, these bandpass SDMs can achieve higher signal-to-noise ratio (SNR) and tonal suppression than those of the original lowpass SDMs.
\end{abstract}


Index Terms - Second order interpolative sigma delta modulators, fractal behavior, limit cycle behavior, chaotic behavior, global stability.

\section{INTRODUCTION}

Sigma delta modulation is a kind of source coding techniques [Janssen, 2003]. An input signal is first sampled at a much higher rate than the Nyquist rate. The most common oversampling ratios are 64, 128 and 256, depending on the applications. Then the sampled signal is subtracted from the output of the interpolative sigma delta modulator (SDM) and filtered via a loop filter. Finally, the loop filter output is quantized to produce the output of the SDM via a very coarse quantizer, such as a single bit quantizer. The block diagram of an interpolative SDM is shown in Figure 1 [Janssen, 2003]. Because of the simple, robust and inexpensive circuit implementation, many systems employ interpolative SDMs to perform analog-to-digital (A/D) conversions [Janssen, 2003].

By modeling the quantizer as a white noise source and properly designing the loop filter, the magnitude of the noise transfer function can be very small at the signal band. This design method is called the noise shaping technique [Janssen, 2003]. However, small magnitude of the noise transfer function at the signal band sometimes does not guarantee a good performance of the SDM, in particular, when the state vectors of the SDM is suffered from divergence and limit cycle problems. This is because the noise shaping technique assumes that the quantization noise is independent of the input of the quantizer. Nevertheless, the quantization noise is input dependent. Hence, the noise shaping technique cannot explain the occurrence of some nonlinear behaviors, such as limit cycle [Hein at al., 1993], fractal [Ashwin, 2003; Davies, 1997; Feely, 1997; Petkov, 1997] and chaotic [Hein, 1993; Schreier, 1994] behaviors, as well as the divergence of the system states when the linearized closed loop transfer function of the SDM is stable [Schreier, 1993; Steiner, 1997]. If a SDM exhibits the divergent behavior for some initial conditions, then when electric shocking occurs, the current state vectors of the SDM will probably be excited to those states that lead to the divergent behavior. In this case, the SDM will be damaged. For some applications, such as audio application, the occurrence of the limit cycle behavior may result to annoying audio tone [Reefman, 2002]. We can see that both the limit cycle and divergent behaviors would cause a degradation of the performance of the SDM, so the occurrence of these behaviors should be avoided. It is well known that limit cycle [Hein at al., 1993] and divergent behaviors [Schreier, 1993; Steiner, 1997] usually occur especially in high order SDMs whereas high order SDMs can produce very small magnitudes of the noise transfer functions at the signal bands. Even for second order lowpass SDMs, these problems still exist [Wang, 1992; Farrell, 1998] when the input magnitudes are at large 
or intermediate levels.

Although some existing control strategies, such as clipping [Reefman, 2002], have been proposed to limit the maximum absolute value of the state variables, it may result to the occurrence of limit cycles. This situation usually occurs when the input signal is very slow time varying and the value of the clipped level is very small. To avoid the occurrence of limit cycles, dithering has been proposed [Magrath, 1995]. However, dithering would increase the circuit complexity and the implementation cost. In this paper, we employ a simple SDM that guarantees both the global stability property and the avoidance of the limit cycle behavior. Here, the global stability property refers to the property of the SDM that the state variables are bounded for all initial conditions in the state space.

It was discussed in [Ashwin, 2003] that if second order bandpass interpolative SDMs exhibit fractal behaviors [Ashwin, 2003], then an invariant set [Ashwin, 2003], [Güntürk, 2004; Schreier at al., 1997; Thao, 2002, 2004] exists for these SDMs. It was also found by [Ashwin, 2003] that some of these SDMs achieve the global stability. On the other hand, it was reported in [Güntürk, 2004; Schreier at al., 1997; Thao, 2002, 2004] that the existence of an invariant set as well as some other conditions will guarantee the global stability property. However, the global stability conditions in [Güntürk, 2004; Schreier at al., 1997; Thao, 2002, 2004] are not satisfied for second order bandpass interpolative SDMs. This is because the SDM studied in [Güntürk, 2004; Schreier at al., 1997; Thao, 2002, 2004] are based on feedbackward structures, in which these results cannot directly be applied to the second order bandpass interpolative SDMs with loop filters having arbitrarily filter coefficients. It was found by [Ho, 2006] that some of second order bandpass interpolative SDMs may exhibit the divergent behavior if the frequency spectrum of the input of the loop filter contains an impulse located at the natural frequency of the loop filter. This implies that the global stability of second order bandpass interpolative SDMs is not universally guaranteed and the global stability of these SDMs is still opened. One of the objectives of this paper is to address this issue. The global stability conditions for these second order bandpass interpolative SDMs is analyzed by the root locus approach [Baird, 1994]. Moreover, we numerically show that these SDMs may also exhibit irregular and conical-like chaotic patterns on the phase plane. For these two behaviors, the global stability property of these SDMs has not been investigated yet.

In this paper, the difference between the fractal and irregular or conical-like chaotic behaviors is also investigated. It was reported in [Ashwin, 2003] that if the filter parameter of a second order bandpass interpolative SDM is within a certain range, then fractal behavior will occur. However, the relationship between the occurrence of the fractal behavior and the magnitude of the input signal has not exploited yet. In this 
paper, we investigate this relationship. The importance of studying this relationship is to provide some guidelines for SDM engineers to operate these SDMs, so that the occurrence of unwanted behaviors can be avoided and high tonal suppression can be obtained.

The outline of this paper is as follows. Notations are introduced in Section II. In Section III, analytical and simulated results are presented. Finally, a conclusion is drawn in Section IV.

\section{NOTATIONS}

We assume that the loop filter is causal and rational with real valued coefficients and a unit delay element multiplied by the numerator of the transfer function. We make this assumption because of the feedback loop configuration. Hence, the transfer function of the second order loop filter can be denoted as:

$$
F(z) \equiv \frac{G z^{-1}\left(1-b z^{-1}\right)}{\left(1-a^{\prime} z^{-1}\right)\left(1-a z^{-1}\right)},
$$

where $a$ and $a^{\prime}$ are the poles, $b$ is the zero and $G$ is related to the DC gain of the loop filter. Since the input signal is oversampled, the input is very slow time varying and we can approximate it as a DC signal. That is, by denoting $u(k)$ as the input of the second order interpolative SDM and $\bar{u}$ as the input step size, we have $u(k) \approx \bar{u}$ for $k \geq 0$. This assumption can be validated via testing the performance of the SDM by using a sinusoidal input with frequency within the signal band $\left[0, \frac{\pi}{R}\right]$, in which $R$ denotes the oversampling ratio [Schreier, 2003]. Denote the output of the loop filter as $y(k)$. The dynamics of this SDM can be described by the following state space equation: $\mathbf{x}(k+1)=\mathbf{A x}(k)+\mathbf{B}(\mathbf{u}(k)-\mathbf{s}(k))$ for $k \geq 0$,

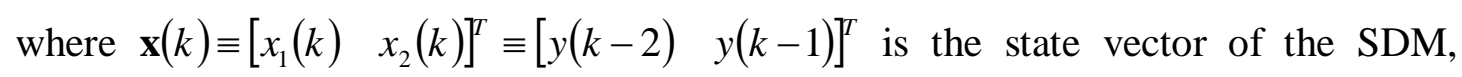
$\mathbf{u}(k) \equiv[u(k-2) u(k-1)]^{T}$ is the vector containing the past two consecutive points from the input signal $u(k)$,

$$
\mathbf{A} \equiv\left[\begin{array}{cc}
0 & 1 \\
-a a^{\prime} & a+a^{\prime}
\end{array}\right]
$$

is the system matrix,

$$
\mathbf{B} \equiv\left[\begin{array}{cc}
0 & 0 \\
-b G & G
\end{array}\right]
$$

is the matrix associated with the input signal and the nonlinearity due to the quantizer,

$$
\mathbf{s}(k) \equiv\left[Q\left(x_{1}(k)\right) \quad Q\left(x_{2}(k)\right)\right]^{T} \text { for } k \geq 0
$$


is the quantized state vector in which the superscript ${ }^{T}$ denotes the transpose operator, and

$$
Q(y) \equiv\left\{\begin{array}{cc}
1 & y \geq 0 \\
-1 & \text { otherwise }
\end{array}\right.
$$

is a single bit quantization function.

\section{MAIN RESULTS}

A. Magnitude response of the loop filter in a second order bandpass interpolative SDM

Now, let us consider the second order bandpass interpolative SDM discussed in [Ashwin, 2003; Davies, 1997; Feely, 1997; Petkov, 1997], where the parameters of the filter are $G=2 \cos \theta, a=e^{j \theta}, a^{\prime}=e^{-j \theta}$ and $b=\frac{1}{2 \cos \theta}$, in which $\theta$ is the natural frequency of the loop filter. Although the bandpass filter is employed, the magnitude response of the bandpass filter is close to that of the lowpass filter when $\theta$ is very close to zero, in particular, when $|\omega| \geq 2 \theta$. Hence, the magnitude response of the lowpass filter can be approximated by the bandpass filter with the natural frequency very close to zero. Here, very close to zero means that $\theta$ is much smaller than $\frac{\pi}{R}$. Figure 2 shows the magnitude responses of a second order bandpass filter with $\theta=0.001$ and a second order lowpass filter with $a=a^{\prime}=1, b=\frac{1}{2}$ and $G=2$. It can be seen from Figure 2 that the magnitude responses of these two filters are almost the same when $|\omega| \geq 2 \theta$.

\section{B. Global stability property of the second order bandpass interpolative SDM}

As discussed in Section I that an invariant set exists for a class of second order bandpass interpolative SDMs [Ashwin, 2003] when this class of SDMs exhibits fractal behaviors. Also, some of these SDMs will be globally stable. However, this property has not been proved in [Ashwin, 2003]. Moreover, the global stability condition has not been investigated yet if fractal behaviors do not occur. In this subsection, we will address the following issues: First, what are the general global stability conditions for these SDMs? Second, do invariant sets exist when these SDMs do not exhibit fractal behavior? Third, will the state vectors move towards these invariant sets when these SDMs do not exhibit fractal behavior? To address the first problem, we have the following result.

\section{Lemma 1}

For the class of second order bandpass interpolative SDMs discussed in [Ashwin, 2003], if the ratio of the output of the SDM to the output of the loop filter tends to zero 
as well as the frequency spectrum of the input of the loop filter contains an impulse located at the natural frequency of the loop filter, then this SDM will diverge.

\section{Proof:}

To prove this lemma, the root locus approach [Baird, 1994] is employed. That is, the quantizer is modeled as an amplifier with variable gain $K$.

As $K \equiv \frac{s(k)}{y(k)}$ and $s(k)=Q(y(k)) \in\{1,-1\} \quad \forall k \geq 0, K>0$. The poles of the linearized closed loop transfer function are:

$$
\lambda_{1}(K)=(1-K) \cos \theta+\sqrt{(K-1)^{2} \cos ^{2} \theta+K-1}
$$

and

$$
\lambda_{2}(K)=(1-K) \cos \theta-\sqrt{(K-1)^{2} \cos ^{2} \theta+K-1} .
$$

If $0<K<1$, then $\operatorname{imag}\left(\lambda_{i}(K)\right) \neq 0$ for $i=1,2$ and it can be checked easily that $\left|\lambda_{i}(K)\right|=\sqrt{1-K}<1$ for $i=1,2$ and $\forall \theta \in[-\pi, \pi]$. For $K=0, \lambda_{1}=e^{j \theta}$ and $\lambda_{2}=e^{-j \theta}$.

For $K \geq 1, \operatorname{imag}\left(\lambda_{i}(K)\right)=0$ for $i=1,2$. It can be checked easily that if $\cos \theta<\frac{1}{2}$ and $K>\frac{2(1-\cos \theta)}{1-2 \cos \theta}$, then $K>1$ and $\lambda_{1}(K)>1$. If $\cos \theta>-\frac{1}{2}$ and $K>\frac{2(\cos \theta+1)}{2 \cos \theta+1}$, then $K>1$ and $\lambda_{2}(K)<-1$. Since $\frac{2(1-\cos \theta)}{1-2 \cos \theta}<\frac{2(\cos \theta+1)}{2 \cos \theta+1}$ for $0>\cos \theta>-\frac{1}{2}$ and vice versa for $\frac{1}{2}>\cos \theta>0$, by combining all these conditions, the stability of the linearized closed loop transfer function is summarized as follows:

If $K>\frac{2(1-\cos \theta)}{1-2 \cos \theta}$ and $\cos \theta<0$, or $K>\frac{2(\cos \theta+1)}{2 \cos \theta+1}$ and $\cos \theta>0$, then the linearized closed loop transfer function will be unstable. If $K=0$, or $\cos \theta=0$ and $K=\frac{2(1-\cos \theta)}{1-2 \cos \theta}=\frac{2(\cos \theta+1)}{2 \cos \theta+1}=2 \quad, \quad$ or $\quad K=\frac{2(1-\cos \theta)}{1-2 \cos \theta} \quad$ and $\cos \theta<0 \quad, \quad$ or $K=\frac{2(\cos \theta+1)}{2 \cos \theta+1}$ and $\cos \theta>0$, then the linearized closed loop transfer function will be marginally stable. Otherwise, the linearized closed loop transfer function will be strictly stable. The stability region is shown in Figure 3.

As $K \equiv \frac{s(k)}{y(k)}$ and $s(k)=Q(y(k)) \in\{1,-1\}, y(k)$ is unbounded if and only if $K \rightarrow 0^{+}$. Hence, the stability analysis can be performed by only considering the case when $K \rightarrow 0^{+}$. It is worth noting that this linear approach is applied even though it is 
employed for the analysis of a nonlinear system. When $K \rightarrow 0^{+}$, the linearized closed loop transfer function is marginally stable. For any bounded input $u(k)$, the input of the loop filter is bounded because $s(k)$ is bounded. As the SDM consists of the marginally stable loop filter, the only possible cause for $y(k)$ being unbounded is the resonance effect. That is, the frequency spectrum of the input of the loop filter contains an impulse located at the natural frequency of the loop filter. This completes the proof.

From the above, we can see that large value of $|y(k)|$ corresponds to small value

of $K$. If $|y(k)|$ is large and $K \rightarrow 0^{+}$, then the linearized closed loop transfer function is marginally stable. If the frequency spectrum of the input of the loop filter contains an impulse located at the natural frequency of the loop filter, then the state vectors will diverge.

As fractal behaviors could be exhibited for a class of second order bandpass interpolative SDMs, we can understand the exhibitions of fractal behaviors from the above root locus analysis. It is worth noting that small values of $|y(k)|$ correspond to large value of $K$ and an unstable linearized closed loop transfer function. Hence, for small values of $|y(k)|$, the state vectors will move outwards from the region around the origin. On the other hand, large values of $|y(k)|$ correspond to small value of $K$ and a strictly stable linearized closed loop transfer function if $K$ does not tend to zero. Hence, for large values of $|y(k)|$, the state vectors will move towards the region around the origin. Hence, the state vectors will move in and out of the region around the origin again and again. This accounts for the occurrence of the fractal and irregular chaotic behaviors.

To investigate whether an invariant set exists for the SDM when the SDM does not exhibit the fractal behavior, we need to recall the definition of an invariant set [Ashwin, 2003], [Güntürk, 2004; Schreier at al., 1997; Thao, 2002, 2004]. Denote a map $G$ which maps from a set $\Gamma$ to itself. That is, $G: \Gamma \rightarrow \Gamma$. If $G(\Gamma)=\Gamma$, then $\Gamma$ is called an invariant set. Denote $\varnothing$ as the empty set.

\section{Lemma 2}

Denote

$$
\Gamma \equiv\left\{\begin{array}{l}
\mathbf{x} \equiv\left[\begin{array}{ll}
x_{1}, & x_{2}
\end{array}\right]^{T}: \mathbf{A} \mathbf{x}+\mathbf{B}(\overline{\mathbf{u}}-Q(\mathbf{x})) \in \Gamma, \mathbf{x}-2\left[Q\left(x_{1}\right), \quad 0\right]^{T} \notin \Gamma, \text { and } \\
{\left[\begin{array}{ll}
-Q(\bar{x})-\bar{x}, & x_{1}
\end{array}\right]^{T} \in \Gamma, \text { where } \bar{x} \equiv x_{2}+2 \cos \theta\left(Q\left(x_{1}\right)-x_{1}\right)+\bar{u}(1-2 \cos \theta) .}
\end{array}\right\} .
$$


Denote $G: \Gamma \rightarrow \Gamma$ such that $G(\mathbf{x}) \equiv \mathbf{A x}+\mathbf{B}(\overline{\mathbf{u}}-Q(\mathbf{x}))$. Suppose that $\Gamma \neq \emptyset$. Then $\Gamma$ is an invariant set under the system map $G$.

Proof:

Since $\forall \mathbf{x} \in \Gamma, G(\mathbf{x})=\mathbf{A x}+\mathbf{B}(\overline{\mathbf{u}}-Q(\mathbf{x})) \in \Gamma$. This implies that $G(\Gamma) \subseteq \Gamma$. $\forall \mathbf{y} \equiv\left[y_{1}, \quad y_{2}\right]^{T} \in \Gamma \quad, \quad$ define $\quad \bar{y} \equiv y_{2}+2 \cos \theta\left(Q\left(y_{1}\right)-y_{1}\right)+\bar{u}(1-2 \cos \theta)$. Denote $\mathbf{x} \equiv\left[-Q(\bar{y})-\bar{y}, \quad y_{1}\right]^{T}$. Then $\mathbf{x} \in \Gamma$ and

$$
\begin{aligned}
& G(\mathbf{x})=\mathbf{A} \mathbf{x}+\mathbf{B}(\overline{\mathbf{u}}-Q(\mathbf{x})) \\
& =\left[y_{1}, \quad Q(\bar{y})+\bar{y}+2 y_{1} \cos \theta+2 \bar{u} \cos \theta-2 Q\left(y_{1}\right) \cos \theta-\bar{u}+Q(-Q(\bar{y})-\bar{y})\right]^{T} . \\
& =\left[y_{1}, \quad Q(\bar{y})+Q(-Q(\bar{y})-\bar{y})+y_{2}\right]^{T}
\end{aligned}
$$

If $\bar{y} \geq 0$, then $Q(\bar{y})=1, Q(-Q(\bar{y})-\bar{y})=-1$ and $G(\mathbf{x})=\mathbf{y}$. If $\bar{y}<0$, then $Q(\bar{y})=-1$, $Q(-Q(\bar{y})-\bar{y})=1$ and $G(\mathbf{x})=\mathbf{y}$. Hence, $\forall \mathbf{y} \in \Gamma, \exists \mathbf{x} \in \Gamma$ such that $G(\mathbf{x})=\mathbf{y}$. This implies that $G(\Gamma) \supseteq \Gamma$. Therefore, $G(\Gamma)=\Gamma$ and $\Gamma$ is an invariant set under the system map $G$. This completes the proof.

There are some conventional approaches to test whether a set of state vectors of a $\mathrm{SDM}$ is an invariant set or not. One of the conventional approaches is based on the geometric property of the set. Although this conventional approach can be applied for those SDMs which exhibit the fractal behavior, because the corresponding set consists of trapezoids, this conventional approach cannot be applied if the shape of the sets is irregular. In fact, we will show numerically at the end of this subsection that a bandpass SDM may exhibit irregular and conical-like chaotic patterns on the phase plane. By applying Lemma 2, we can conclude that these sets are invariant sets because Lemma 2 is satisfied for these cases.

To investigate whether some state vectors, which are initially not inside the invariant set $\Gamma$, will eventually move towards the invariant set $\Gamma$ if the invariant set $\Gamma$ exists, the injective property of $G$ has to be investigated. However, in general, the existence of invariant sets does not imply that the corresponding system maps are injective. Consider the following counter-example: Define $f:[0,1] \rightarrow[0,1]$ as a map such that $f(x)=\bmod (2 x, 1)$, where mod is denoted as the modulo operator. Obviously, $[0,1]$ is an invariant set under the map $f$, but $f$ is not injective.

\section{Lemma 3}

Suppose that $\Gamma \neq \varnothing$. Then $G$ is bijective.

Proof:

From Lemma 2, we have $G(\Gamma)=\Gamma$. This implies that $G$ is surjective. To show that $G$ is injective, denote $\mathbf{x}^{1} \equiv\left[\begin{array}{ll}x_{1}^{1}, & x_{2}^{1}\end{array}\right]^{T}$ and $\mathbf{x}^{2} \equiv\left[\begin{array}{ll}x_{1}^{2}, & x_{2}^{2}\end{array}\right]^{T}$. Assume that $\mathbf{x}^{1}, \mathbf{x}^{2} \in \Gamma$, 
International Journal of Bifurcation and Chaos

$\mathbf{x}^{1} \neq \mathbf{x}^{2} \quad$ and $\quad G\left(\mathbf{x}^{1}\right)=G\left(\mathbf{x}^{2}\right) \quad . \quad G\left(\mathbf{x}^{1}\right)=G\left(\mathbf{x}^{2}\right) \quad$ implies $\quad$ that $x_{2}^{1}=x_{2}^{2}$ and $Q\left(x_{1}^{1}\right)-Q\left(x_{1}^{2}\right)=x_{1}^{1}-x_{1}^{2}$. Since $\mathbf{x}^{1} \neq \mathbf{x}^{2}$ and $x_{2}^{1}=x_{2}^{2}, x_{1}^{1} \neq x_{1}^{2}$. This implies that $Q\left(x_{1}^{1}\right) \neq Q\left(x_{1}^{2}\right)$ and $x_{1}^{2}=x_{1}^{1}-2 Q\left(x_{1}^{1}\right)$. As $\mathbf{x}^{2}=\mathbf{x}^{1}-2\left[Q\left(x_{1}^{1}\right), \quad 0\right]^{T} \notin \Gamma$, this contradicts to $\mathbf{x}^{2} \in \Gamma$. This implies that $G$ is injective. Hence, $G$ is bijective. This completes the proof.

\section{Lemma 4}

Define $H: \mathfrak{R}^{2} \rightarrow \mathfrak{R}^{2}$ as a map that $H(\mathbf{x}) \equiv \mathbf{A x}+\mathbf{B}(\overline{\mathbf{u}}-Q(\mathbf{x}))$. Then $H$ is surjective.

Proof:

Denote $\quad \mathbf{x} \equiv\left[\begin{array}{ll}x_{1}, & x_{2}\end{array}\right]^{T} \quad, \quad \mathbf{y} \equiv\left[\begin{array}{ll}y_{1}, & y_{2}\end{array}\right]^{T} \quad$ and $\bar{y} \equiv y_{2}+2 \cos \theta\left(Q\left(y_{1}\right)-y_{1}\right)+\bar{u}(1-2 \cos \theta) . \forall \mathbf{y} \in \mathfrak{R}^{2}$, let $x_{2}=y_{1}$ and $x_{1}=-Q(\bar{y})-\bar{y}$. Then $\mathbf{x} \in \mathfrak{R}^{2}$ and $\mathbf{y}=H(\mathbf{x})$. Hence, $H$ is surjective. This completes the proof.

\section{Lemma 5}

$H$ is not injective.

Proof:

Denote $\mathbf{x}^{1} \equiv\left[x_{1}^{1}, \quad x_{2}^{1}\right]^{T}$ and consider the case when $\left|x_{1}\right|<2$. Define

$$
\begin{aligned}
& \mathbf{x}^{2} \equiv\left[x_{1}^{2},\right.\left.x_{2}^{2}\right]^{T} \equiv \mathbf{x}^{1}-2\left[Q\left(x_{1}^{1}\right), \quad 0\right]^{T} . \text { Then } \\
& H\left(\mathbf{x}^{1}\right)=\left[x_{2}^{1}, \quad 2 \cos x_{2}^{1}-x_{1}^{1}+(2 \cos \theta-1) \bar{u}+Q\left(x_{1}^{1}\right)-2 \cos \theta Q\left(x_{2}^{1}\right)\right]^{T}
\end{aligned}
$$

and

$$
H\left(\mathbf{x}^{2}\right)=\left[x_{2}^{1}, \quad 2 \cos x_{2}^{1}-x_{1}^{1}+2 Q\left(x_{1}^{1}\right)+(2 \cos \theta-1) \bar{u}+Q\left(x_{1}^{1}-2 Q\left(x_{1}^{1}\right)\right)-2 \cos \theta Q\left(x_{2}^{1}\right)\right]^{T} .
$$

Since $\left|x_{1}\right|<2, Q\left(x_{1}^{1}-2 Q\left(x_{1}^{1}\right)\right)=-Q\left(x_{1}^{1}\right)$ and $H\left(\mathbf{x}^{1}\right)=H\left(\mathbf{x}^{2}\right)$. This implies that $H$ is not injective. This completes the proof.

If $\mathbf{x}(0) \in \Gamma$, since $\mathbf{x}(1)=\mathbf{A} \mathbf{x}(0)+\mathbf{B}(\overline{\mathbf{u}}-Q(\mathbf{x}(0)))=G(\mathbf{x}(0))$, from Lemma 2, we have $\mathbf{x}(1) \in \Gamma$. Similarly, we can conclude that $\mathbf{x}(k) \in \Gamma$ for $k \geq 0$. Hence, the SDM is locally stable. Define $\Gamma_{S} \equiv\left\{\mathbf{x} \in \Gamma:\left|x_{1}\right|<2\right\}$ and $\Gamma_{R} \equiv G\left(\Gamma_{S}\right)$. As $\Gamma_{S} \subseteq \Gamma$, from Lemma 2, we have $\Gamma_{R} \subseteq \Gamma \quad$. Assume that $\Gamma_{S} \neq \varnothing \quad$ Define $\widetilde{\Gamma}_{1} \equiv\left\{\mathbf{x}-2\left[Q\left(x_{1}\right), \quad 0\right]^{T}: \mathbf{x} \equiv\left[x_{1}, \quad x_{2}\right]^{T} \in \Gamma_{S}\right\}$. Since $\Gamma_{S} \neq \varnothing, \tilde{\Gamma}_{1} \neq \varnothing$ and $\Gamma \neq \varnothing$. From

Lemma 2, we can conclude that $\tilde{\Gamma}_{1} \cap \Gamma=\emptyset$. From Lemma 4 and Lemma 5, we can 
conclude that $\forall \mathbf{y} \in \Gamma_{R} \quad, \quad \exists \mathbf{x}_{1} \in \Gamma_{S} \quad$ and $\exists \mathbf{x}_{2} \in \tilde{\Gamma}_{1} \quad$ such that $\quad \mathbf{x}^{1} \neq \mathbf{x}^{2}$ and $G\left(\mathbf{x}_{1}\right)=H\left(\mathbf{x}_{2}\right)=\mathbf{y} \in \Gamma_{R}$. This implies that if $\mathbf{x}(0) \in \tilde{\Gamma}_{1} \backslash \Gamma$, then $\mathbf{x}(1)=H(\mathbf{x}(0)) \in \Gamma_{R}$ and $\mathbf{x}(k) \in \Gamma$ for $k \geq 1$. Or in other words, there exists some state vectors, which are initially not in the invariant set $\Gamma$, but they will eventually move towards the invariant set $\Gamma$ if $\Gamma_{S} \neq \varnothing$.

Suppose that $\Gamma_{S} \neq \varnothing$. Define $\tilde{\Gamma}_{n+1}$ such that $H\left(\tilde{\Gamma}_{n+1}\right)=\tilde{\Gamma}_{n}$ for $n \geq 1$. Then the state vectors in $\tilde{\Gamma}_{n}$ will move to $\tilde{\Gamma}_{n-1}$, and then move to $\tilde{\Gamma}_{n-2}$, and continue to move until they move to $\widetilde{\Gamma}_{1}$, and eventually reach $\Gamma_{R}$ and stay inside $\Gamma$ forever.

Now, we investigate whether all the state vectors, which are initially not in the invariant set $\Gamma$, will eventually move towards the invariant set $\Gamma$, when the invariant set $\Gamma$ exists and the frequency spectrum of the input of the loop filter does not contain an impulse located at the natural frequency of the loop filter. If the frequency spectrum of the input of the loop filter does not contain an impulse located at the natural frequency of the loop filter, then the state vectors will be bounded. Denote $\Lambda$ as the corresponding bounded set. Denote the set $\Lambda \backslash \Gamma \backslash \bigcup_{\forall n \geq 1} \tilde{\Gamma}_{n}$ as $\AA$.

\section{Lemma 6}

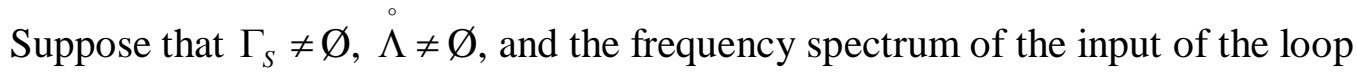
filter does not contain an impulse located at the natural frequency of the loop filter. Then $\stackrel{\circ}{\Lambda}$ is an invariant set under the system map $H$.

Proof:

Since $\Gamma_{S} \neq \varnothing$, this implies that $\widetilde{\Gamma}_{1} \neq \varnothing$. As $H$ is surjective, this further implies that $\tilde{\Gamma}_{n} \neq \varnothing$ for $n \geq 1$. Since the frequency spectrum of the input of the loop filter does not contain an impulse located at the natural frequency of the loop filter, the state

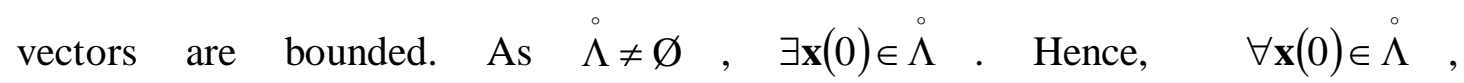
$H(\mathbf{x}(0))=\mathbf{A x}(0)+\mathbf{B}(\overline{\mathbf{u}}-Q(\mathbf{x}(0))) \in \Lambda$. However, $H(\mathbf{x}(0)) \notin \Gamma \backslash \bigcup_{\forall n \geq 1} \tilde{\Gamma}_{n}$. Otherwise, it 


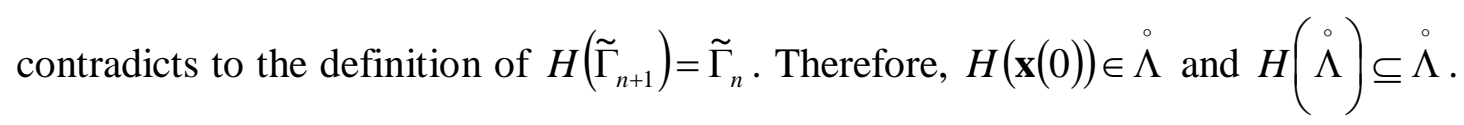
$\forall \mathbf{y} \in \stackrel{\circ}{\Lambda}$, as $H$ is surjective and the state vectors are bounded, $\exists \mathbf{x} \in \Lambda$ such that $H(\mathbf{x})=\mathbf{y}$. However, $\mathbf{x} \notin \Gamma \backslash \bigcup_{\forall n \geq 1} \tilde{\Gamma}_{n}$. Otherwise, it contradicts to the definition of

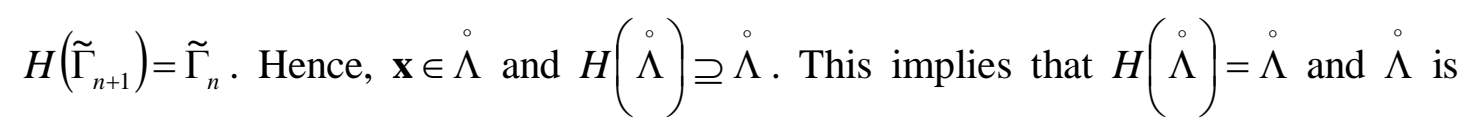
an invariant set under the system map $H$. This completes the proof.

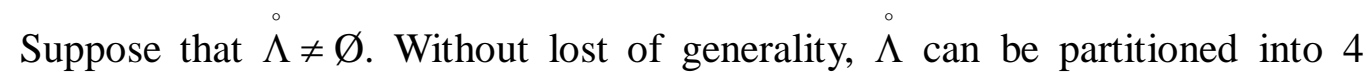
different subsets, denoted as $\Lambda_{i}$ for $i=1,2,3,4$, such that $\forall \mathbf{x}_{i}, \mathbf{x}_{j} \in \Lambda_{k} Q\left(\mathbf{x}_{i}\right)=Q\left(\mathbf{x}_{j}\right)$ and $Q\left(\Lambda_{i}\right) \neq Q\left(\Lambda_{j}\right)$ for $i \neq j, \Lambda_{i} \cap \Lambda_{j}=\varnothing$ for $i \neq j$, and $\bigcup_{i=1}^{4} \Lambda_{i}=\stackrel{\circ}{\text {. }}$

\section{Theorem 1}

If $\Gamma_{S} \neq \varnothing$ and the frequency spectrum of the input of the loop filter does not

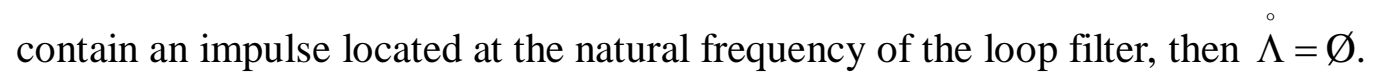

Proof:

From Lemma 1 , we see that if $K \neq 0$, or $\cos \theta<0$ and $K<\frac{2(1-\cos \theta)}{1-2 \cos \theta}$, or $\cos \theta>0$ and $K<\frac{2(\cos \theta+1)}{2 \cos \theta+1}$, or $\cos \theta=0$ and $K<2$, then the linearized closed loop transfer function of the SDM will be strictly stable. This implies that the SDM is strictly stable for $0<K<\frac{4}{3}$. This further implies that if $|y(k)|>\frac{3}{4}$ and the frequency spectrum of the input of the loop filter does not contain an impulse located at the natural frequency of the loop filter, then the SDM will be strictly stable and larger values of $|y(k)|$ will move to a region around the origin. Since $\Gamma_{S} \neq \varnothing, \tilde{\Gamma}_{n} \neq \varnothing$ for $n \geq 1$. Suppose that $\stackrel{\circ}{\Lambda} \neq \varnothing$. If $\exists \mathbf{x}_{1} \equiv\left[\begin{array}{ll}x_{1}^{1}, & x_{2}^{1}\end{array}\right]^{T}, \mathbf{x}_{2} \equiv\left[\begin{array}{ll}x_{1}^{2}, & x_{2}^{2}\end{array}\right]^{T} \in \Lambda_{i}$ such that $\mathbf{x}_{2}=\mathbf{x}_{1}-2\left[Q\left(x_{1}^{1}\right), \quad 0\right]^{T}$, then the trajectories corresponding to these two initial conditions will move to a region around the origin. Hence, we can just consider the case that $\mathbf{x}_{1} \in \Lambda_{i}$ and $\mathbf{x}_{2} \in \Lambda_{j}$ for $i \neq j$. Suppose that $\mathbf{x}_{1}+\left[\begin{array}{ll}\Delta x, & 0\end{array}\right]^{T} \in \Lambda_{i}$ for $\forall \Delta x \in(-\delta, \delta)$ and $\mathbf{x}_{2}+[\Delta x, \quad 0]^{T} \in \Lambda_{j}$ for 
$\forall \Delta x \in(-\delta, \delta)$, where $\delta$ is the length of the neighborhood of $\mathbf{x}_{1}$ and $\mathbf{x}_{2}$. Since $H\left(\mathbf{x}_{1}+\left[\begin{array}{ll}\Delta x, & 0\end{array}\right]^{T}\right)=H\left(\mathbf{x}_{1}\right)-\left[\begin{array}{ll}0, & \Delta x\end{array}\right]^{T} \quad, \quad H\left(\mathbf{x}_{2}+\left[\begin{array}{ll}\Delta x, & 0\end{array}\right]^{T}\right)=H\left(\mathbf{x}_{2}\right)-\left[\begin{array}{ll}0, & \Delta x\end{array}\right]^{T} \quad$ and $H\left(\mathbf{x}_{1}\right)=H\left(\mathbf{x}_{2}\right)$, the regions after the system map are overlapped. However, this contradicts to the definition of an invariant set with the system map having the property $|\operatorname{det}(\mathbf{A})|=1$. Hence, $\stackrel{\circ}{\Lambda}=\varnothing$. This completes the proof.

Theorem 1 implies that, if $\Gamma_{S} \neq \varnothing$ and the frequency spectrum of the input of the loop filter does not contain an impulse located at the natural frequency of the loop filter, then for all the initial conditions that are initially not in the invariant set $\Gamma, \exists k_{0} \in \mathrm{Z}^{+}$

such that $\mathbf{x}\left(k_{0}\right) \in \widetilde{\Gamma}_{1}$. That means, the SDM is globally stable and the state vectors will eventually move to the invariant set. To illustrate the theorem, the black, blue, cyan, yellow, green, magenta and red regions in Figure $4 \mathrm{a}$ and $4 \mathrm{~b}$ show the sets $\tilde{\Gamma}_{6}, \tilde{\Gamma}_{5}, \tilde{\Gamma}_{4}$,

$\tilde{\Gamma}_{3}, \tilde{\Gamma}_{2}, \tilde{\Gamma}_{1}$ and $\Gamma_{R}$, respectively.

Corollary 1

Define

$L_{1} \equiv \sum_{j=0}^{M-1} \sin (M-1-j) \theta\left(2 \cos \theta\left(\bar{u}-Q\left(x_{2}(j)\right)\right)-\left(\bar{u}-Q\left(x_{1}(j)\right)\right)\right)$,

$L_{2} \equiv \sum_{j=0}^{M-1} \cos (M-1-j) \theta\left(2 \cos \theta\left(\bar{u}-Q\left(x_{2}(j)\right)\right)-\left(\bar{u}-Q\left(x_{1}(j)\right)\right)\right)$,

$\tilde{L}_{1} \equiv \tilde{x}_{1}(0) \cos M \theta+\tilde{x}_{2}(0) \sin M \theta$

and

$\tilde{L}_{2} \equiv-\tilde{x}_{1}(0) \sin M \theta+\tilde{x}_{2}(0) \cos M \theta$.

Denote $\mathbf{T} \equiv\left[\begin{array}{cc}1 & 0 \\ \cos \theta & \sin \theta\end{array}\right]$. Then $\mathbf{T}^{-1}$ exists for bandpass filters. Define the transformed state vectors as $\widetilde{\mathbf{x}}(k)=\mathbf{T}^{-1} \mathbf{x}(k)$. Then $\forall \mathbf{x}(0) \in \Lambda \backslash \Gamma, \exists M \in Z^{+}$such that $\mathbf{x}(M) \in \Gamma$. Also, if $\forall \mathbf{y} \in \Gamma \quad\|\tilde{\mathbf{x}}(0)\|_{2}>\left\|\mathbf{T}^{-1} \mathbf{y}\right\|_{2}$, then $\exists M \in Z^{+}$such that $\left(L_{1}+\tilde{L}_{1}\right)^{2}+\left(L_{2}+\tilde{L}_{2}\right)^{2}<\tilde{L}_{1}^{2}+\tilde{L}_{2}^{2}$

\section{Proof:}

Since $\sin \theta$ is zero only for lowpass or highpass filters, $\mathbf{T}^{-1}$ exists for bandpass 
filters. According to Theorem 1, as $\stackrel{\Lambda}{\Lambda}=\varnothing, \forall \mathbf{x}(0) \in \Lambda \backslash \Gamma, \exists M \in Z^{+}$such that $\mathbf{x}(M) \in \Gamma$. Define $\widehat{\mathbf{A}} \equiv\left[\begin{array}{cc}\cos \theta & \sin \theta \\ -\sin \theta & \cos \theta\end{array}\right]$. Hence, $\mathbf{A}=\mathbf{T} \widehat{\mathbf{A}} \mathbf{T}^{-1}$ and

$$
\mathbf{x}(M)=\mathbf{A}^{M} \mathbf{x}(0)+\sum_{j=0}^{M-1} \mathbf{A}^{M-1-j} \mathbf{B}(\overline{\mathbf{u}}-Q(\mathbf{x}(j))) .
$$

This implies that

$$
\tilde{\mathbf{x}}(M)=\widehat{\mathbf{A}}^{M} \tilde{\mathbf{x}}(0)+\sum_{j=0}^{M-1} \widehat{\mathbf{A}}^{M-1-j} \mathbf{T}^{-1} \mathbf{B}(\overline{\mathbf{u}}-Q(\mathbf{x}(j)))=\left[\begin{array}{c}
\tilde{x}_{1}(0) \cos M \theta+\tilde{x}_{2}(0) \sin M \theta+L_{1} \\
-\tilde{x}_{1}(0) \sin M \theta+\tilde{x}_{2}(0) \cos M \theta+L_{2}
\end{array}\right]
$$

and $\|\tilde{\mathbf{x}}(M)\|_{2}^{2}-\|\tilde{\mathbf{x}}(0)\|_{2}^{2}=\left(L_{1}+\tilde{L}_{1}\right)^{2}+\left(L_{2}+\tilde{L}_{2}\right)^{2}-\tilde{L}_{1}^{2}-\tilde{L}_{2}^{2}$. If $\forall \mathbf{y} \in \Gamma \quad\|\tilde{\mathbf{x}}(0)\|_{2}>\left\|\mathbf{T}^{-1} \mathbf{y}\right\|_{2}$,

then $\exists M \in Z^{+}$such that $\|\tilde{\mathbf{x}}(M)\|_{2}<\|\tilde{\mathbf{x}}(0)\|_{2}$. Hence, $\exists M \in Z^{+}$such that $\left(L_{1}+\tilde{L}_{1}\right)^{2}+\left(L_{2}+\tilde{L}_{2}\right)^{2}<\tilde{L}_{1}^{2}+\tilde{L}_{2}^{2}$. This completes the proof.

Corollary 1 provides information on how the initial condition moves towards the invariant set $\Gamma$.

Beside, some interesting phenomena are found. First of all, fractal patterns are not the only type of chaotic patterns exhibited in the phase plane. The SDM may exhibit irregular and conical-like chaotic patterns. Figure $5 \mathrm{a}$ and $5 \mathrm{~b}$ show the phase portraits when $\mathbf{x}(0)=\mathbf{0}, \bar{u}=-0.3, \theta=\cos ^{-1}(0.158532)$, and $\mathbf{x}(0)=\mathbf{0}, \bar{u}=0.5, \theta=0.1$, respectively. Figure $5 \mathrm{c}$ shows the transformed phase portrait $\widetilde{\mathbf{x}}(k)$ when $\mathbf{x}(0)=\mathbf{0}$, $\bar{u}=-1$ and $\theta=0.01$. It can be seen from Figure 5a, 5b and $5 \mathrm{c}$ that fractal, irregular and conical-like chaotic patterns are exhibited on the phase plane, respectively. However, no matter what type of chaotic patterns is, an invariant set exists. By dividing the phase portraits, shown in Figure $6 a, 6 c$ and $6 e$, into four subportraits, it can be seen, respectively, in Figure $6 \mathrm{~b}, 6 \mathrm{~d}$ and $6 \mathrm{f}$, that the union of the mapped regions generates the original phase portraits with the same outer boundaries.

The second interesting phenomenon is that $\Gamma \backslash \Gamma_{S}=\varnothing$ when the SDM exhibits fractal patterns, while $\Gamma \backslash \Gamma_{S} \neq \varnothing$ when the SDM exhibits irregular and conical-like chaotic patterns. To understand this phenomenon, note that the fractal patterns are confined within two trapezoids, the co-ordinates of the outer four corners of these two trapezoids not including the co-ordinates on the $x_{2}$ axis are:

$$
\begin{aligned}
& \mathbf{P}_{1}=\left[\begin{array}{ll}
(2 \cos \theta-1)(\bar{u}-1) & \left(4 \cos ^{2} \theta-1\right)(\bar{u}-1)
\end{array}\right]^{T}, \\
& \mathbf{P}_{2}=\left[\begin{array}{ll}
2 \cos \theta(\bar{u}+1)+1-\bar{u} & \left(4 \cos ^{2} \theta-1\right)(\bar{u}+1)
\end{array}\right]^{T},
\end{aligned}
$$




$$
\mathbf{P}_{3}=\left[\begin{array}{lll}
(2 \cos \theta-1)(\bar{u}+1) & \left(4 \cos ^{2} \theta-1\right)(\bar{u}+1)
\end{array}\right]^{T}
$$

and

$$
\mathbf{P}_{4}=\left[\begin{array}{ll}
2 \cos \theta(\bar{u}-1)-\bar{u}-1 \quad\left(4 \cos ^{2} \theta-1\right)(\bar{u}-1)
\end{array}\right]^{T},
$$

respectively. We will show in Subsection IIID that the conditions for exhibiting fractal behaviors are $|\cos \theta|<\frac{1}{2}$ and $|\bar{u}|<\min \left(1, \frac{1+2 \cos \theta}{1-2 \cos \theta}\right)$, and the absolute values of the first co-ordinate of these four state vectors is bounded by 2 . Note that these two trapezoids are in $\Gamma_{S}$. Hence, $\Gamma=\Gamma_{S}$ and $\Gamma \backslash \Gamma_{S}=\varnothing$. However, this property does not hold for the cases when irregular and conical-like chaotic patterns are exhibited on the phase plane. The red regions shown in Figure $7 \mathrm{a}$ and $7 \mathrm{c}$ correspond to $\Gamma_{S}$, while the blue region shown in Figure $7 \mathrm{c}$ corresponds to $\Gamma \backslash \Gamma_{S}$. The red regions shown in Figure $7 \mathrm{~b}$ and $7 \mathrm{~d}$ correspond to $\Gamma_{R}$, while the blue region shown in Figure $7 \mathrm{~d}$ corresponds to $\Gamma \backslash \Gamma_{R}$. It can be seen in Figure $7 \mathrm{a}$ and $7 \mathrm{~b}$ that there is no blue region in the phase portraits, which implies that $\Gamma \backslash \Gamma_{S}=\varnothing$ when the SDMs exhibit fractal patterns.

C. Conditions for the second order bandpass interpolative SDM exhibiting limit cycle behavior

The necessary and sufficient conditions for the class of second order bandpass interpolative SDMs discussed in [Ashwin, 2003] exhibiting the limit cycle behaviors are presented below.

Suppose that there exists $M \geq 1$ such that $\mathbf{I}-\mathbf{A}^{M}$ is invertible, where $\mathbf{I}$ denotes the identity matrix. Denote $\|\cdot\|_{\infty}$ as the infinity-norm operator,

$$
\begin{aligned}
& \mathbf{x}_{0}^{*} \equiv\left(\mathbf{I}-\mathbf{A}^{M}\right)^{-1}\left(\sum_{j=0}^{M-1} \mathbf{A}^{M-1-j} \mathbf{B}(\overline{\mathbf{u}}-\mathbf{s}(j))\right), \\
& \mathbf{x}_{i+1}^{*} \equiv \mathbf{A} \mathbf{x}_{i}^{*}+\mathbf{B}(\overline{\mathbf{u}}-\mathbf{s}(i)) \text { for } i=0,1, \cdots, M-2,
\end{aligned}
$$

and

$$
\hat{\mathbf{x}}_{i}(k) \equiv \mathbf{T}^{-1}\left(\mathbf{x}(k M+i)-\mathbf{x}_{i}^{*}\right) \text { for } k \geq 0 \text { and } i=0,1, \cdots, M-1 .
$$

The following lemma describes the necessary and sufficient relationships among the periodicity of the output sequence, the behavior of the trajectory and the set of the initial conditions generating periodic output sequence.

\section{Lemma 7}

The following three statements are equivalent:

i) $\exists M \in Z^{+}$such that $\forall i \geq 0 \quad \mathbf{s}(M+i)=\mathbf{s}(i)$. 
ii) $\exists M \in Z^{+}$such that $\widehat{\mathbf{x}}_{i}(k+1)=\widehat{\mathbf{A}}^{M} \widehat{\mathbf{x}}_{i}(k)$ for $k \geq 0$ and for $i=0,1, \cdots, M-1$.

iii) $\exists M \in Z^{+} \quad$ such that $\quad \mathbf{x}(0) \in \Sigma_{M} \equiv\left\{\mathbf{x}(0):\left\|\mathbf{T}^{-1}\left(\mathbf{x}(i)-\mathbf{x}_{i}^{*}\right)\right\| \leq\left\|\mathbf{x}_{i}^{*}\right\|_{\infty}\right\} \quad$ for $i=0,1, \cdots, M-1$.

Proof:

The proof can be worked out using the techniques discussed in [Ling, 2003].

Statement (ii) of Lemma 7 implies that the transformed trajectories are circular. Since $i=0,1, \cdots, M-1$, there are $M$ circles centered at the origin with radii $\left\|\hat{\mathbf{x}}_{i}(0)\right\|_{2}$. By transforming back to the original state trajectories, there are $M$ ellipses centered at $\mathbf{x}_{i}^{*}$ for $i=0,1, \cdots, M-1$ with the size depended on $\|\mathbf{x}(i)\|_{2}$ and the orientation depended on $\theta$. Moreover, from statement (i) of Lemma 7, we can see that the output sequence is periodic with period $M$. Furthermore, from statement (iii) of Lemma 7, we can see that the shape of the set of the initial conditions is elliptic.

There are three main important implications and two interesting phenomena that can be accounted from Lemma 7. The first important implication of Lemma 7 is that for a given initial condition in the trapezoids, Lemma 7 provides information to test whether a limit cycle occurs or not. This can be done by checking whether the given initial condition is inside the ellipses of the fractal patterns or not. If it is inside the ellipses, then a limit cycle occurs, and vice versa.

The second important implication of Lemma 7 is to estimate the periodicity of the limit cycle. Since the periodicity is defined based on the behavior over an infinite amount of time, but only a finite number of observable output bits are obtained from practical situations, in general, it is not trivial to check whether an output sequence is periodic or not. Even though the output sequence is periodic, it is not easy to estimate its periodicity. However, according to Lemma 7, the periodicity of the output sequence can be estimated by counting the number of the ellipses exhibiting on the phase plane or counting the number of the elliptical sets of initial conditions.

The third important implication of Lemma 7 is to provide information whether a limit cycle is stable or not. If a given initial condition is strictly inside an elliptical set of initial conditions, a small perturbation of the initial condition will give rise to a similar elliptic trajectory pattern and the same periodic output sequence as well. Hence, the corresponding trajectory for the limit cycle is regarded as locally stable. However, if the initial condition is on the boundary of the elliptical set, a small perturbation of the initial condition will give rise to a very different dynamical behavior and output sequence. In this case, the trajectory for the limit cycle is regarded as locally unstable. 
There are two interesting phenomena that can be explained by Lemma 7. First, when $M=1, \mathbf{x}_{0}{ }^{*}$ is on the diagonal line. Since the output sequence is constant, all the state vectors are in the same quadrant. Hence, the trajectory and the set of initial conditions are confined either in quadrant I or quadrant III, and cannot cut across the principle axes. For $M \geq 1$, by downsampling the output sequence by $M$, the output sequence becomes constant. Hence, the trajectory of each ellipse and each elliptical set of initial conditions also cannot cut across the principle axes.

Second, the occurrence of periodic output sequence does not imply the occurrence of periodic state variables [Ling, 2003]. In general the state variables are periodic if $\theta$ is a rational multiple of $\pi$.

D. Conditions for the second order bandpass interpolative SDM exhibiting fractal

\section{behavior}

Now, we present the results for the class of second order bandpass interpolative SDMs discussed in [Ashwin, 2003] which exhibits fractal behaviors. The conditions for the occurrence of fractal behaviors are presented below.

\section{Lemma 8}

If

$$
|\cos \theta|<\frac{1}{2}
$$

$|\bar{u}|<\min \left(1, \frac{1+2 \cos \theta}{1-2 \cos \theta}\right)$

$\mathbf{s}(k)$ are aperiodic and

$$
\mathbf{x}(0) \in \Gamma \backslash \bigcup_{M \geq 1} \Sigma_{M}
$$

then

$$
\mathbf{x}(k) \in \Gamma \backslash \bigcup_{M \geq 1} \Sigma_{M}, \text { for } k \geq 0 .
$$

Proof:

It can be proved using the approach discussed in [Ashwin, 2003].

Although this lemma can be proved using the approach discussed in [Ashwin, 2003], the effects of the input step size and the initial conditions to the exhibition of elliptic fractal patterns have not been explored in [Ashwin, 2003] yet. Lemma 8 addresses these issues. We can see from Lemma 8 that elliptic fractal patterns will occur when the filter parameter satisfies the condition stated in equation (21), the input step size is kept below the bound defined in equation (22) and the initial condition satisfies equation (23). Note that when the magnitude of $\bar{u}$ is larger than the bound defined in equation (22), or when the filter parameters are not in the range defined by equation (21) 
or when the initial conditions are not in the set defined in equation (23) but still in the trapezoids, then Lemma 8 will not be satisfied and elliptic fractal pattern will not be exhibited in the phase plane.

The importance of Lemma 7 and Lemma 8 is that they provide information for SDM designers to operate the SDMs so that the occurrences of limit cycle and fractal behaviors can be avoided. Hence, annoying audio tones will not be observed if these SDMs are employed for audio application [Reefman, 2002].

\section{E. Performance of the second order bandpass interpolative SDM}

In the following simulations, we assume that $R=64$ because it is the most common oversampling ratio employed in audio application [Reefman, 2002]. As discussed in Subsection IIIA, the natural frequency of a second order bandpass interpolative SDM should be small, we choose $\theta=0.001$, which is $2.0372 \%$ of the signal bandwidth $\frac{\pi}{R}$. In order to compare the performances of the second order lowpass and bandpass interpolative SDMs, the second order lowpass interpolative SDM with $a=a^{\prime}=1, b=\frac{1}{2}$ and $G=2$ are set because the magnitude response of this lowpass SDM is close to that of the bandpass SDM. We first employ $\mathbf{x}(0)=\mathbf{0}$ for simulations because "initially at rest" is the most common practical situations. Then, we check if the conditions for exhibiting limit cycles and fractal behaviors stated in Section IIIC and Section IIID, respectively, are satisfied or not. If none of these conditions is satisfied, then we will employ this initial condition. Otherwise, we generate another initial condition randomly and check it again. We repeat this checking procedure for no more than 100 iterations. Note that only less than 100 iterations are checked because this can prevent trapping into a dead loop of the generation of the initial conditions.

In order to compare the performance of the lowpass and the bandpass SDMs, tonal suppression and SNR are used as the criteria. Tonal suppression reflects the ability of the avoidance of annoying audio tones and SNR reflects the reconstruction errors of the $\mathrm{A} / \mathrm{D}$ and $\mathrm{D} / \mathrm{A}$ conversions. We define the tonal suppression as:

$$
T S\left(\omega_{0}\right) \equiv \frac{\left|S\left(\omega_{0}\right)\right|}{\max _{\omega \in(0, \pi) \backslash\left\{\omega_{0}\right\}}|S(\omega)|} .
$$

Figure $8 \mathrm{a}$ and Figure $8 \mathrm{~b}$ show the tonal suppression of the lowpass SDM, denoted as $T S_{\text {lowpass }}\left(\omega_{0}\right)$, and that of the bandpass SDM, denoted as $T S_{\text {bandpass }}\left(\omega_{0}\right)$, respectively.

Figure $8 \mathrm{c}$ shows the ratio of the improvement of the tonal suppression, that is 
$\frac{T S_{\text {bandpass }}\left(\omega_{0}\right)-T S_{\text {lowpass }}\left(\omega_{0}\right)}{T S_{\text {lowpass }}\left(\omega_{0}\right)}$. It can be seen from Figure $8 \mathrm{c}$ that there are many positive spikes, which implies that the bandpass SDM has higher tonal suppression than the lowpass SDM for most frequencies and input magnitudes. Besides, Figure 9 shows the magnitude spectra of the output sequences of the lowpass and bandpass SDMs when the DC input with the step size $\bar{u}=0.7$ is applied under the zero initial condition $\mathbf{x}(0)=\mathbf{0}$. It can be seen from Figure 9 that there are impulses in the magnitude spectrum of the output sequence of the lowpass SDM, which demonstrates that the lowpass SDM exhibits the limit cycle behavior. On the other hand, the bandpass SDM operates normally.

The definition of the SNR adopt in this paper is based on that defined in [Schreier, 2003]. Figure 10 shows the SNRs of the lowpass and bandpass SDMs under the same oversampling ratios, that is, $R=64$, and operating conditions. That is, we assume that the frequency of the sinusoidal input is $\frac{2}{3}$ of the signal bandwidth $\frac{\pi}{R}$. According to Figure 10, we can see that the lowpass SDM can only achieve an average of $66.1751 \mathrm{~dB}$ for an input magnitude less than or equal to 1.06, and 9.2952dB for that between 1.07 and 2. On the other hand, the bandpass SDM can achieve an average of $80.7934 \mathrm{~dB}$ for an input magnitude less than or equal to 1.16 , and $12.0046 \mathrm{~dB}$ for that between 1.17 and 2 by using our checking procedures. Hence, the bandpass SDM have an average of $14.6183 \mathrm{~dB}$ and $2.7094 \mathrm{~dB}$ improvements for low and high input magnitudes, respectively, as well as an increasing the input stability margin of 0.1 . These results show that the bandpass SDM with the natural frequency very close to zero can achieve a higher SNR than that of the lowpass SDM, and our derived conditions for checking the exhibition of limit cycle and fractal behaviors are useful for operating the bandpass SDM.

\section{CONCLUSION}

In this paper, we propose to replace a second order lowpass interpolative SDM by a second order bandpass interpolative SDM with the natural frequency of the loop filter very close to zero. If the natural frequency of the bandpass SDM is smaller than the value which depends on the oversampling ratio, then the magnitude response of the bandpass SDM will be close to that of the lowpass SDM. The main advantages of employing the bandpass SDM are that the global stability of the SDM can be guaranteed if the frequency spectrum of the input of the loop filter does not contain an impulse located at the natural frequency of the loop filter. Moreover, the conditions for the occurrence of limit cycle and fractal behaviors are derived, so these unwanted 
behaviors can be avoided accordingly. Simulation results show that the bandpass SDM can achieve higher SNR and tonal suppression than that of the lowpass SDM.

\section{ACKNOWLEDGEMENT}

The work obtained in this paper was supported by a research grant from Queen Mary, University of London.

\section{REFERENCES}

[1] Ashwin, P., Fu, X. C. \& Deane, J. [2003] "Properties of the invariant disk packing in a model bandpass sigma-delta modulator," International Journal of Bifurcations and Chaos, 13, 631-641.

[2] Baird, R. T. \& Fiez, T. S. [1994] "Stability analysis of high-order delta-sigma modulation for ADC's," IEEE Transactions on Circuits and Systems-II: Analog and Digital Signal Processing, 41, 59-62.

[3] Davies, A. C. \& Petkov, G. P. [1997] "Zero-input oscillation bounds in a bandpass $\Sigma \Delta$ modulator," Electronics Letters, 33, 28-29.

[4] Farrell, R. \& Feely, O. [1998] "Bounding the integrator outputs of second-order sigma-delta modulators," IEEE Transactions on Circuits and Systems-II: Analog and Digital Signal Processing, 45, 691-702.

[5] Feely, O. [1997] "A tutorial introduction to non-linear dynamics and chaos and their application to sigma-delta modulators," International Journal of Circuit Theory and Applications, 25, 347-367.

[6] Güntürk, C. S. \& Thao, N. T. [2004] "Refined error analysis in second-order $\Sigma \Delta$ modulation with constant inputs," IEEE Transactions on Information Theory, 50, 839-860.

[7] Hein, S. [1993] "Exploiting chaos to suppress spurious tones in general double-loop $\Sigma \Delta$ modulators," IEEE Transactions on Circuits and Systems-II: Analog and Digital Signal Processing, 40, 651-659.

[8] Hein, S. \& Zakhor, A. [1993] "On the stability of sigma delta modulators," IEEE Transactions on Signal Processing, 41, 2322-2348.

[9] Ho, C. Y. F., Ling, B. W. K. \& Reiss, J. D. [2006] "Stability of sinusoidal responses of marginally stable bandpass sigma delta modulators," International Journal of Circuit Theory and Applications, 34, 593-605.

[10] Janssen, E. \& Reefman, D. [2003] "Super-audio CD: an introduction," IEEE Signal Processing Magazine, 20, 83-90.

[11] Ling, B. W. K., Tam, P. K. S. \& Yu, X. [2003] "Step response of a second-order digital filter with two's complement arithmetic," IEEE Transactions on Circuits and Systems-I: Fundamental Theory and Applications, 50, 510-522. 
[12] Magrath, A. J. \& Sandler, M. B. [1995] "Efficient dithering of sigma-delta modulators with adaptive bit flipping," Electronics Letters, 31, 846-847.

[13] Petkov, G. P. \& Davies, A. C. [1997] "Constraints on constant-input oscillations of a bandpass sigma-delta modulator structure," International Journal of Circuit Theory and Applications, 25, 393-405.

[14] Reefman, D. \& Janssen, E. [2002] "Signal processing for direct stream digital: a tutorial for digital sigma delta modulation and 1-bit digital audio processing," Philips Research, Eindhoven, White Paper.

[15] Schreier, R. [1993] "An empirical study of high-order single-bit delta-sigma modulators," IEEE Transactions on Circuits and Systems-II: Analog and Digital Signal Processing, 40, 461-466.

[16] Schreier, R. [1994] "On the use of chaos to reduce idle-channel tones in delta-sigma modulators," IEEE Transactions on Circuits and Systems-I: Fundamental Theory and Applications, 41, 539-547.

[17] Schreier, R., Goodson, M. V., \& Zhang, B. [1997] "An algorithm for computing convex positively invariant sets for delta-sigma modulators," IEEE Transactions on Circuits and Systems - I: Fundamental Theory and Applications, 44, 38-44.

[18] Schreier, R. [2003] The delta-sigma modulators toolbox version 6.0, Analog Devices Inc..

[19] Steiner, P. \& Yang, W. [1997] “A framework for analysis of high-order sigma-delta modulators," IEEE Transactions on Circuits and Systems-II: Analog and Digital Signal Processing, 44, 1-10.

[20] Thao, N. T. [2002] "MSE behavior and centroid function of $m$ th-order asymptotic $\Sigma \Delta$ modulators," IEEE Transactions on Circuits and Systems-II: Analog and Digital Signal Processing, 49, 86-100.

[21] Thao, N. T. [2004] "The tiling phenomenon in $\Sigma \Delta$ modulation," IEEE Transactions on Circuits and Systems - I: Regular Papers, 51, 1365-1378.

[22] Wang, H. [1992] "A geometric view of $\Sigma \Delta$ modulations," IEEE Transactions on Circuits and Systems - II: Analog and Digital Signal Processing, 39, 402-405.

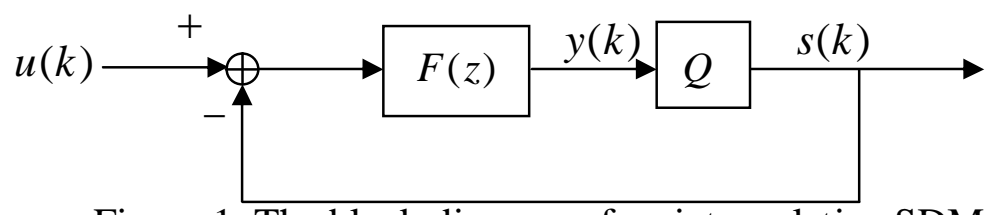

Figure 1. The block diagram of an interpolative SDM. 


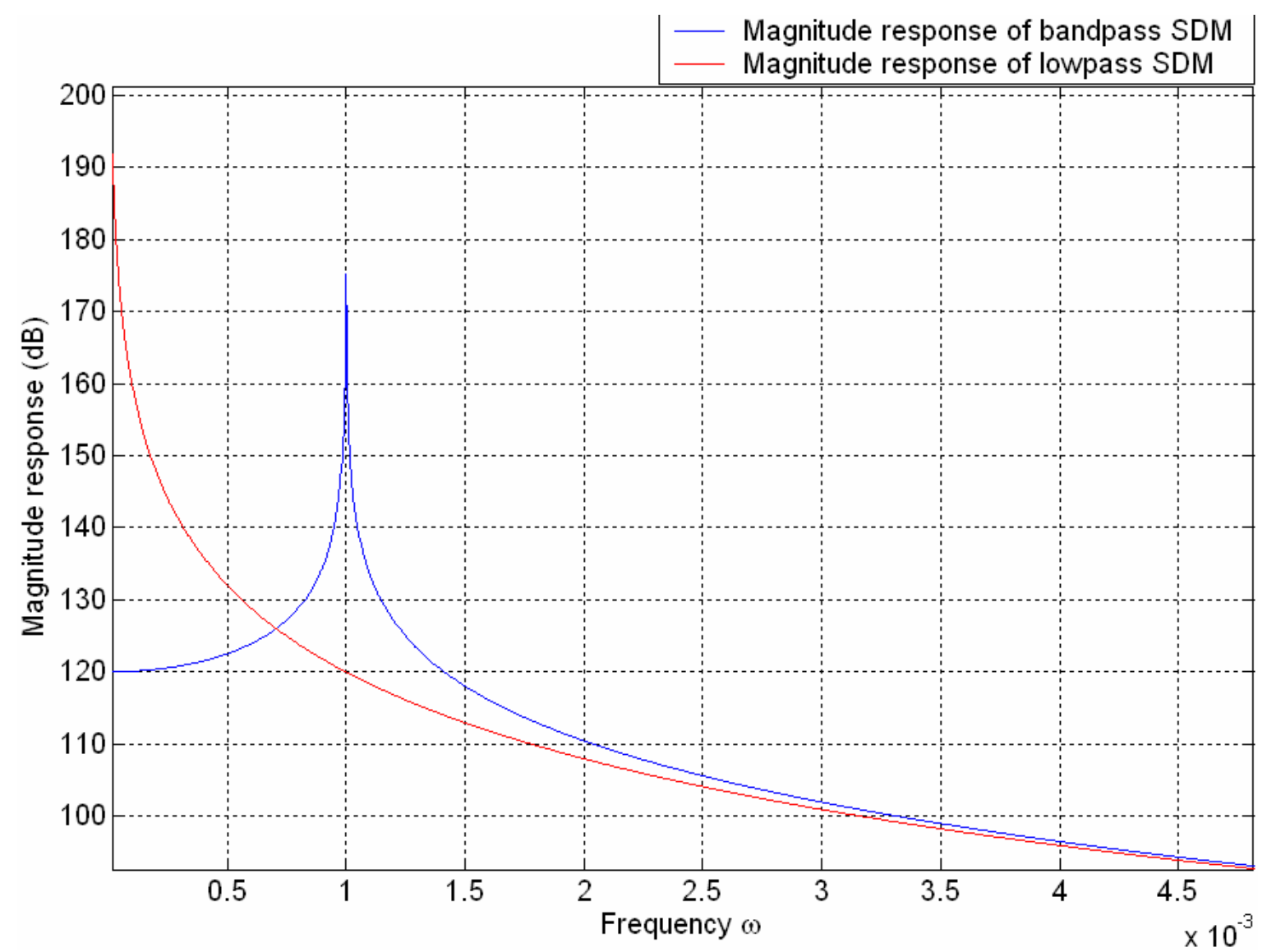

Figure 2. Magnitude responses of both the lowpass and bandpass SDMs.
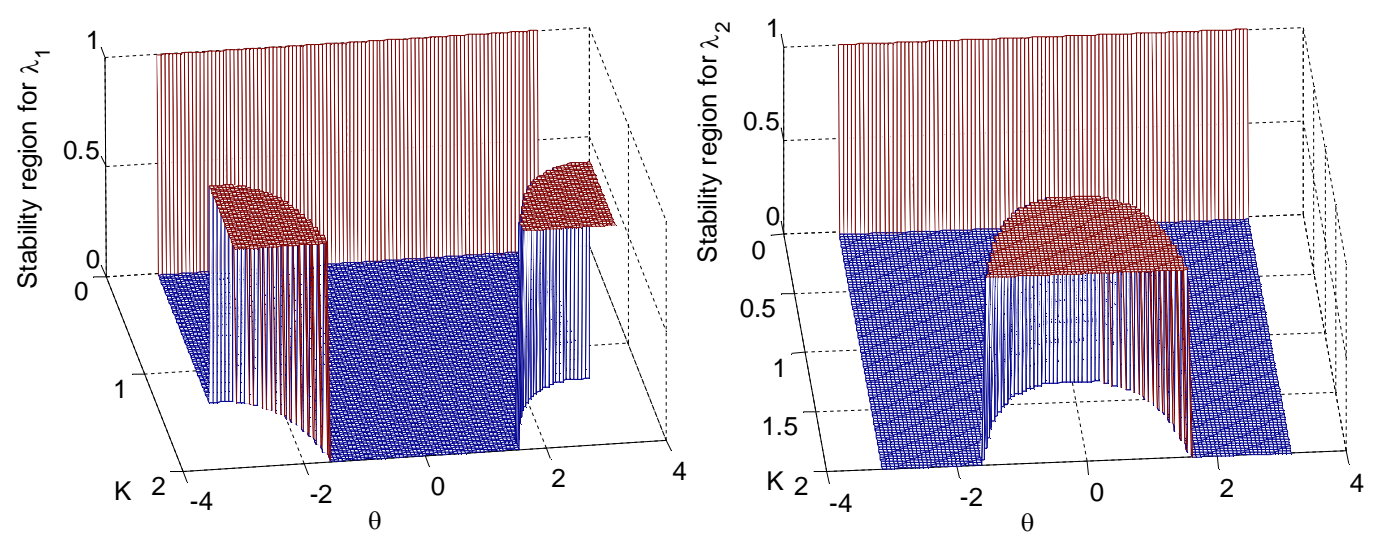

Figure 3. Stability region of the linearized closed loop transfer function of the second order bandpass interpolative SDM (The red region refers to the unstable region and the blue region refers to the strictly stable region.). 
International Journal of Bifurcation and Chaos

(a)

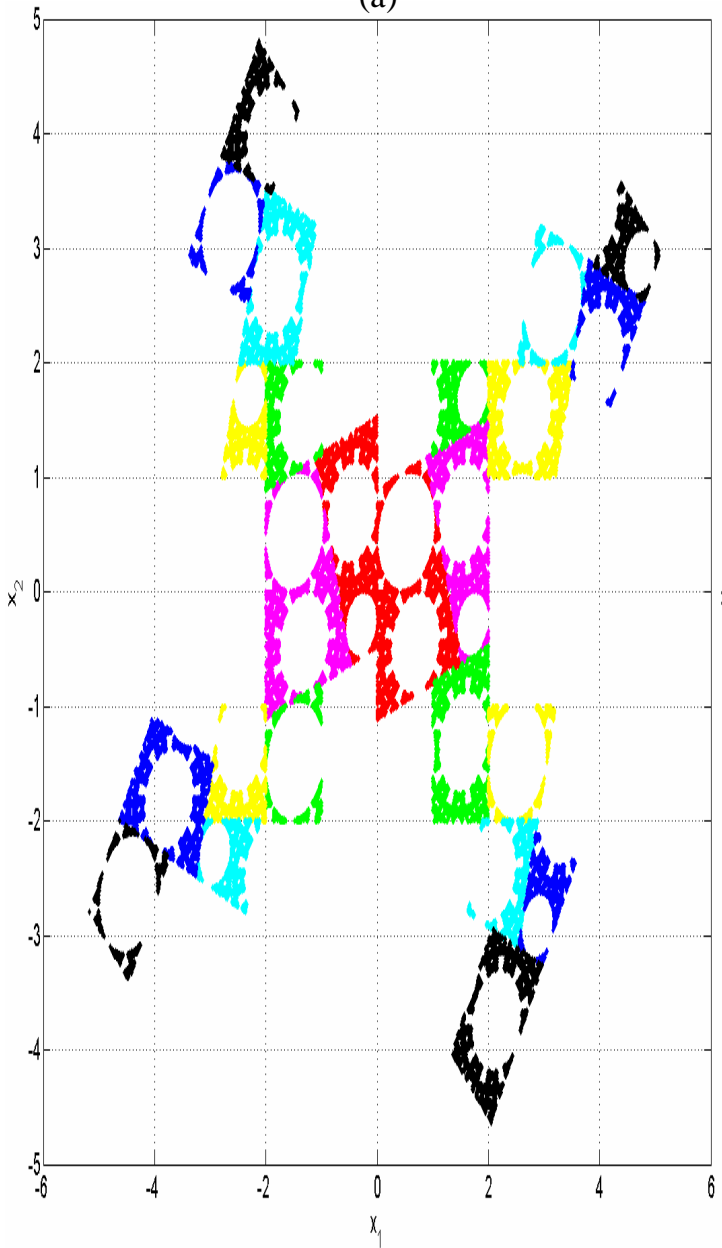

(b)

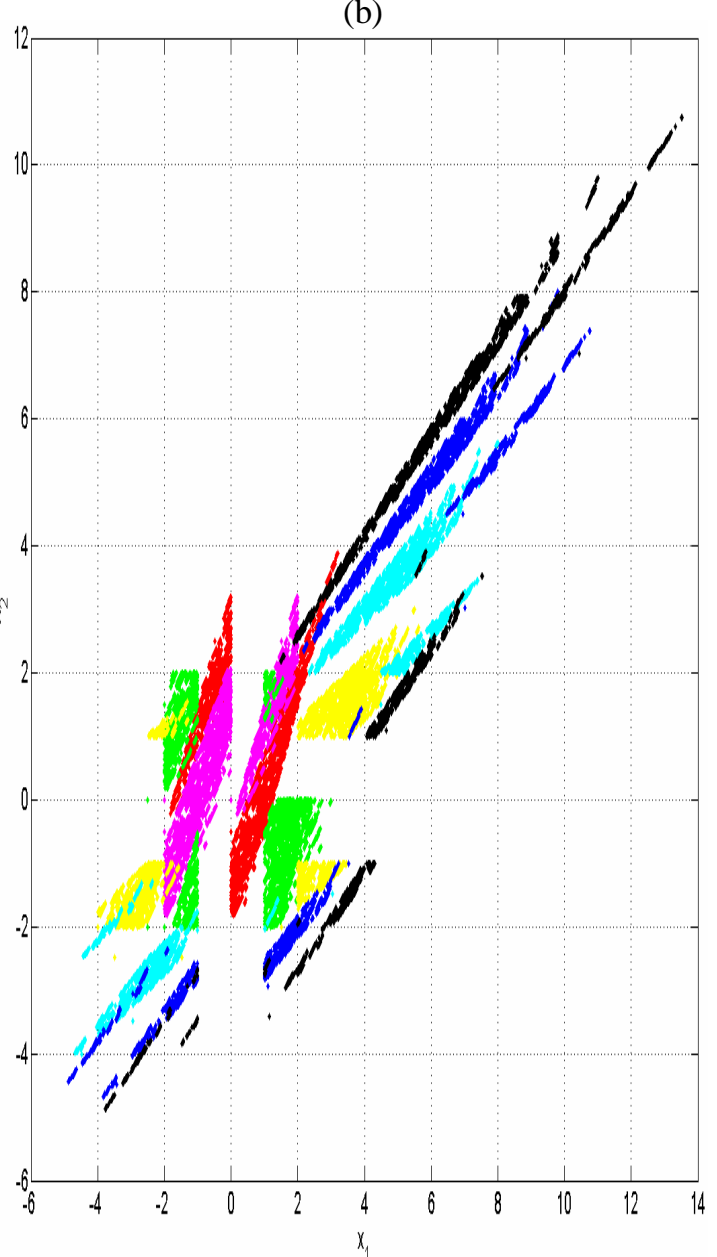

Figure 4. Diagrams showing how the state vectors move towards an invariant set when (a) fractal and (b) irregular chaotic patterns occur.
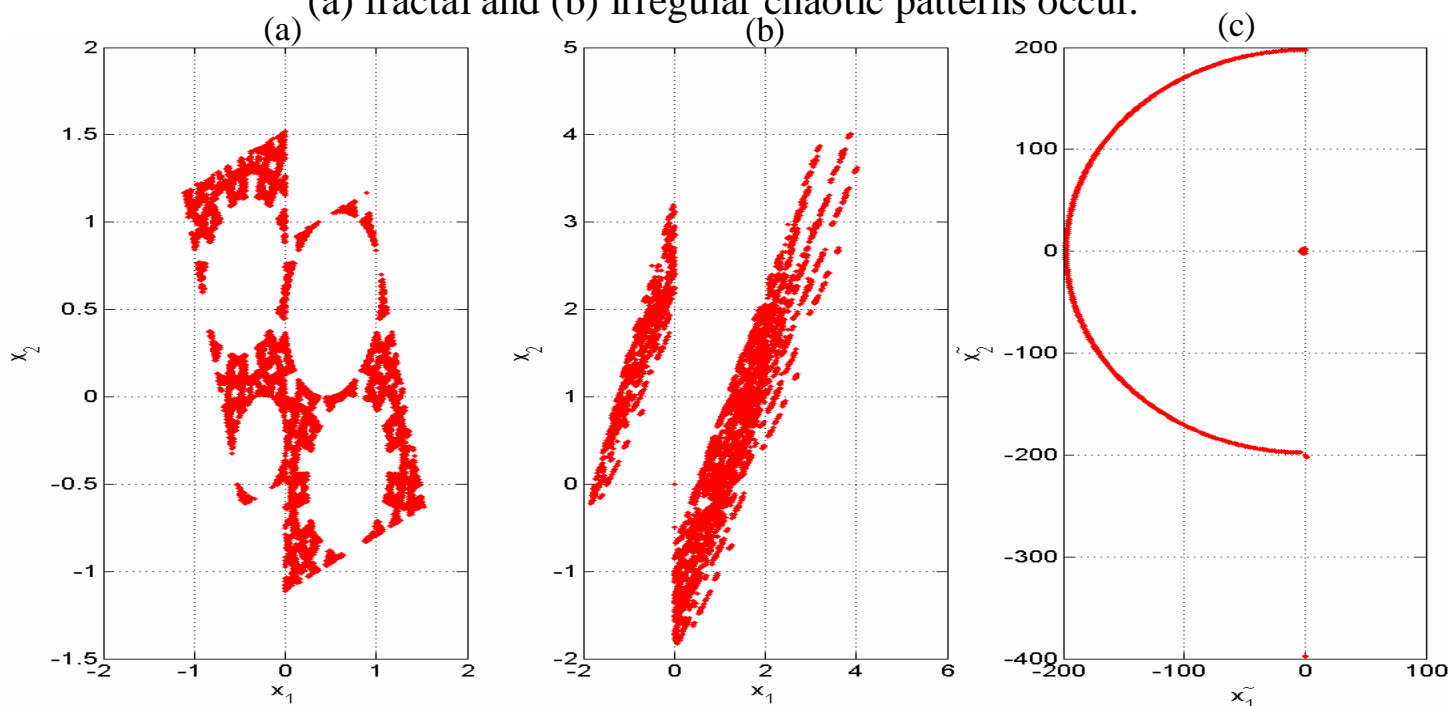

Figure 5. Phase portraits of the SDMs when (a) fractal and (b) irregular chaotic patterns occur. (c) Transformed phase portrait of the SDM when a conical-like chaotic pattern occurs. 
International Journal of Bifurcation and Chaos

(a)

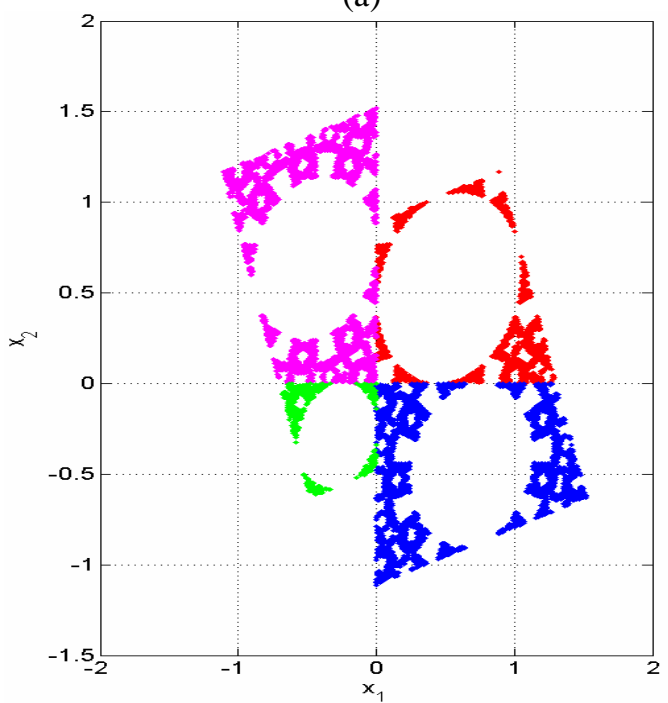

(c)
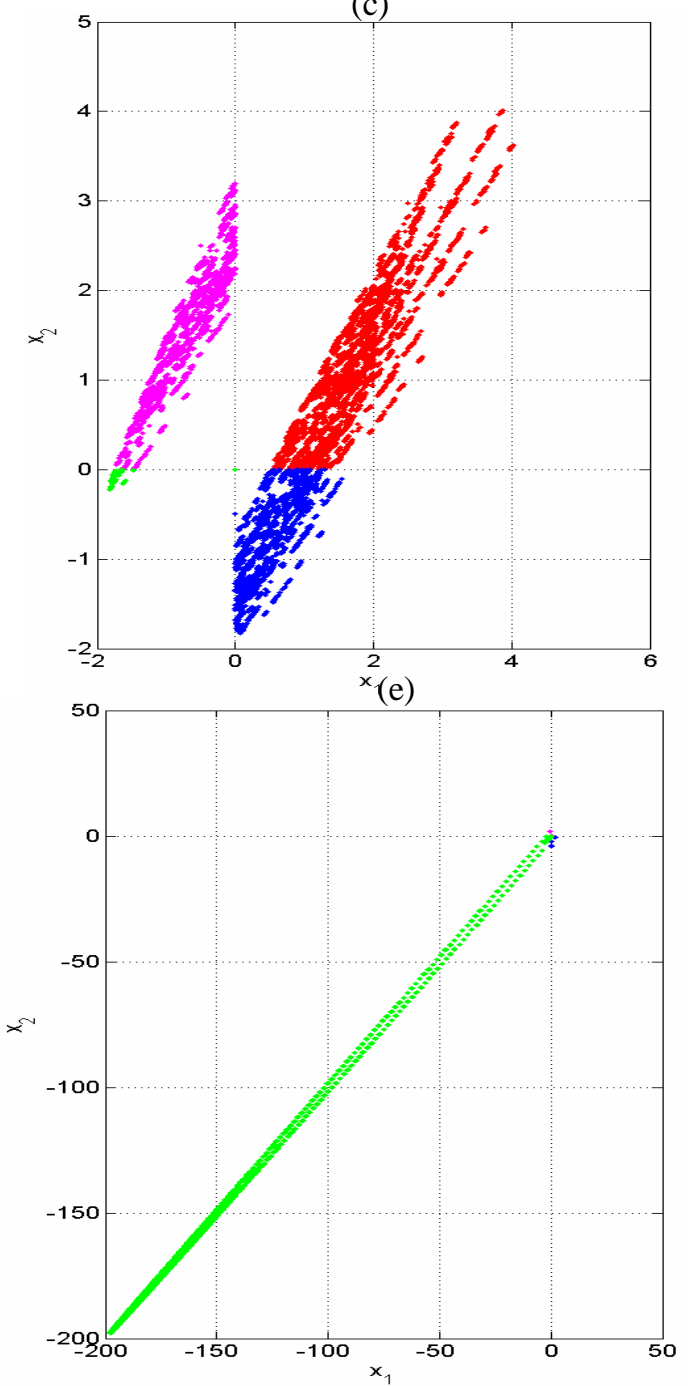

(b)

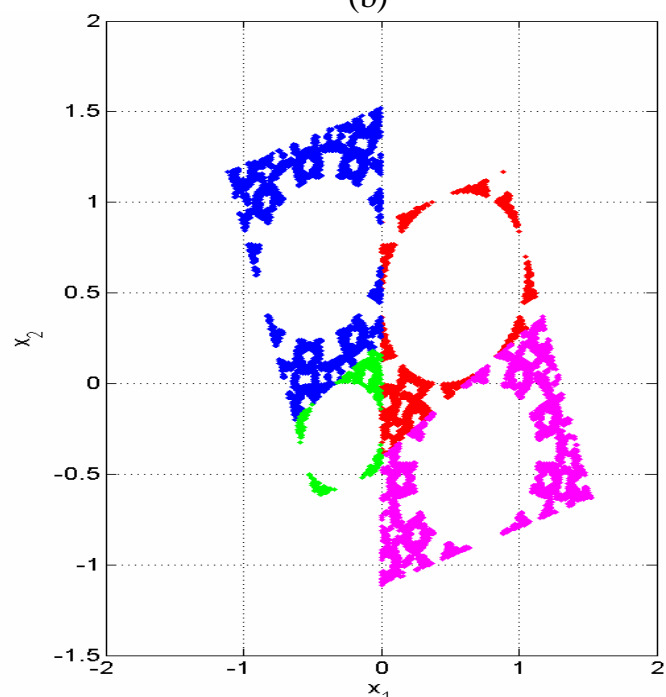

(d)
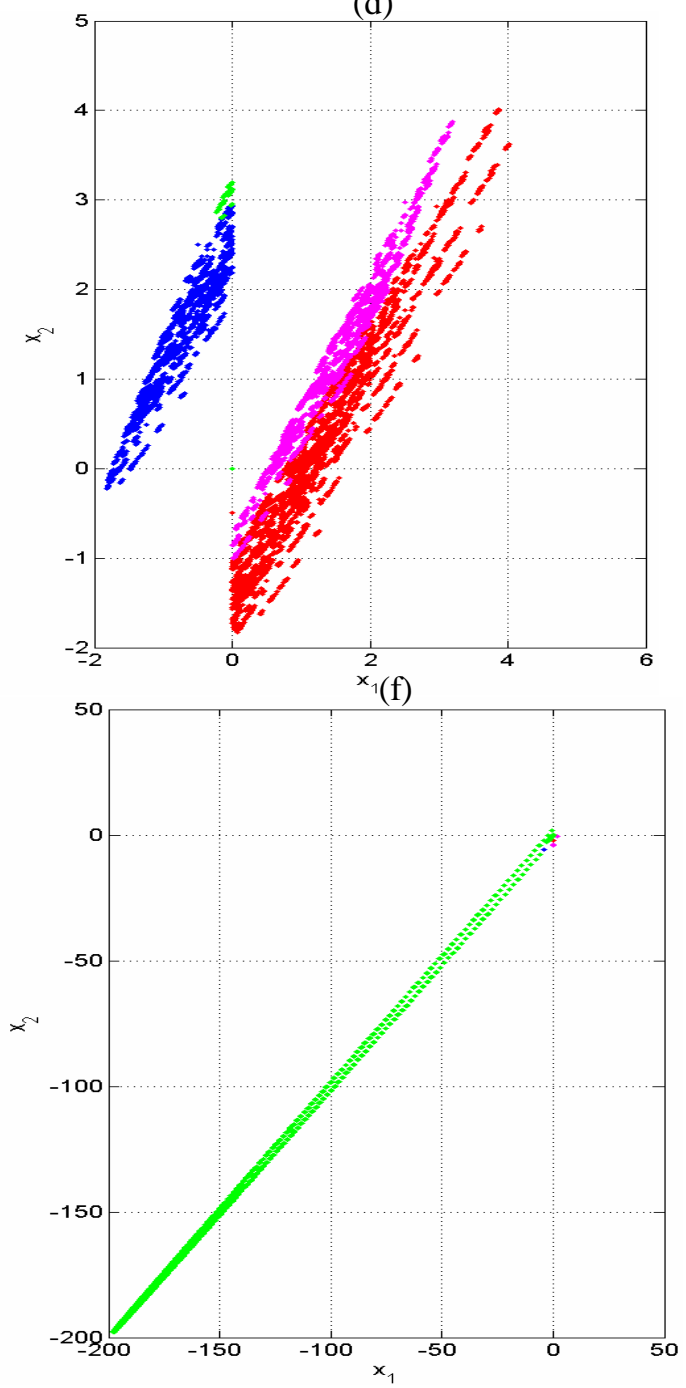

Figure 6. Phase portraits when (a) fractal, (c) irregular and (e) conical-like chaotic patterns occur. (b), (d) and (f) are the corresponding mapped phase portraits. 
International Journal of Bifurcation and Chaos

(a)

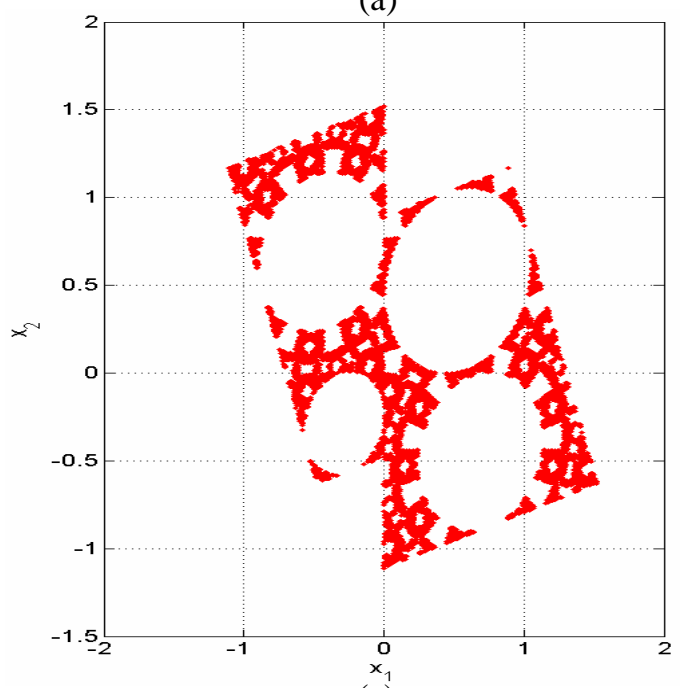

$(c)$

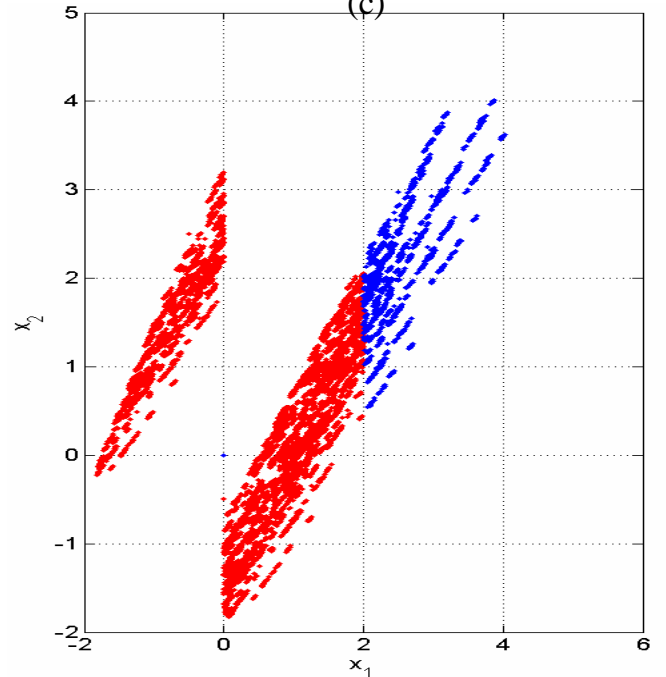

(b)

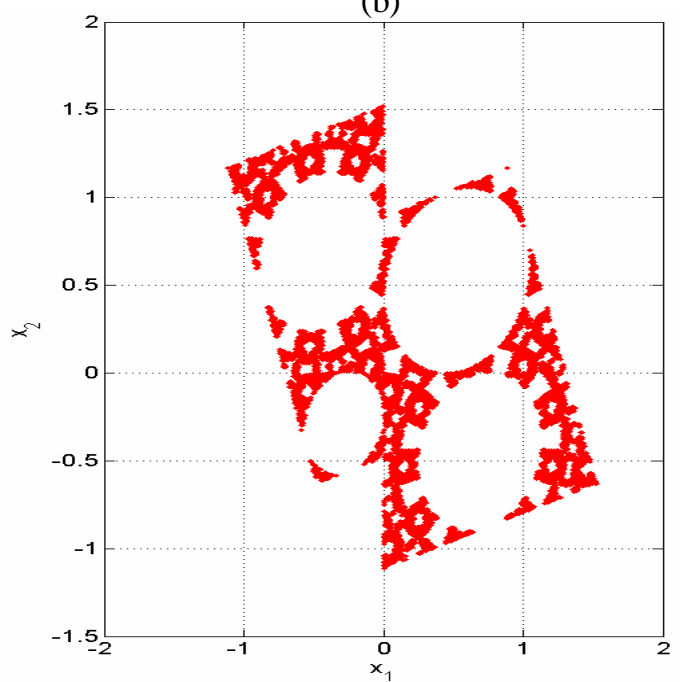

(d)

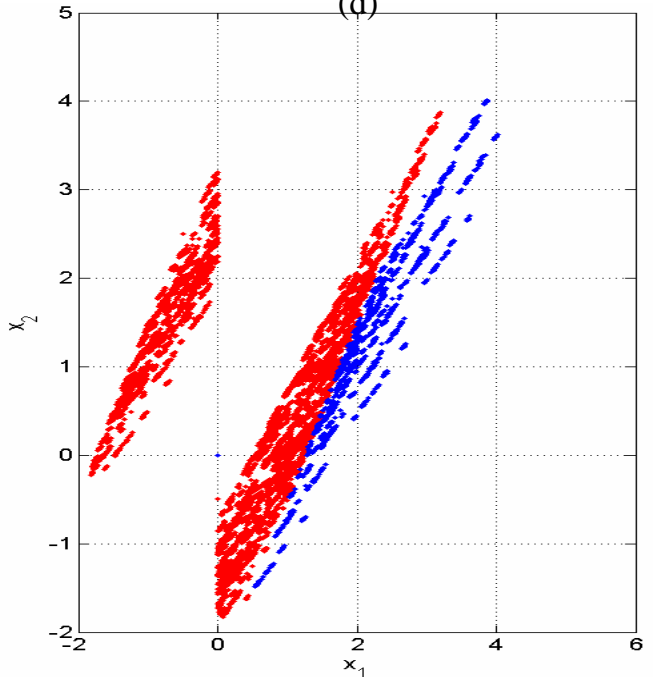

Figure 7. (a) $\Gamma_{S}$ and (b) $\Gamma_{R}$ when a fractal pattern occurs. (c) $\Gamma_{S}$ and $\Gamma \backslash \Gamma_{S}$, and (d) $\Gamma_{R}$ and $\Gamma \backslash \Gamma_{R}$ when an irregular chaotic pattern occurs. 
International Journal of Bifurcation and Chaos

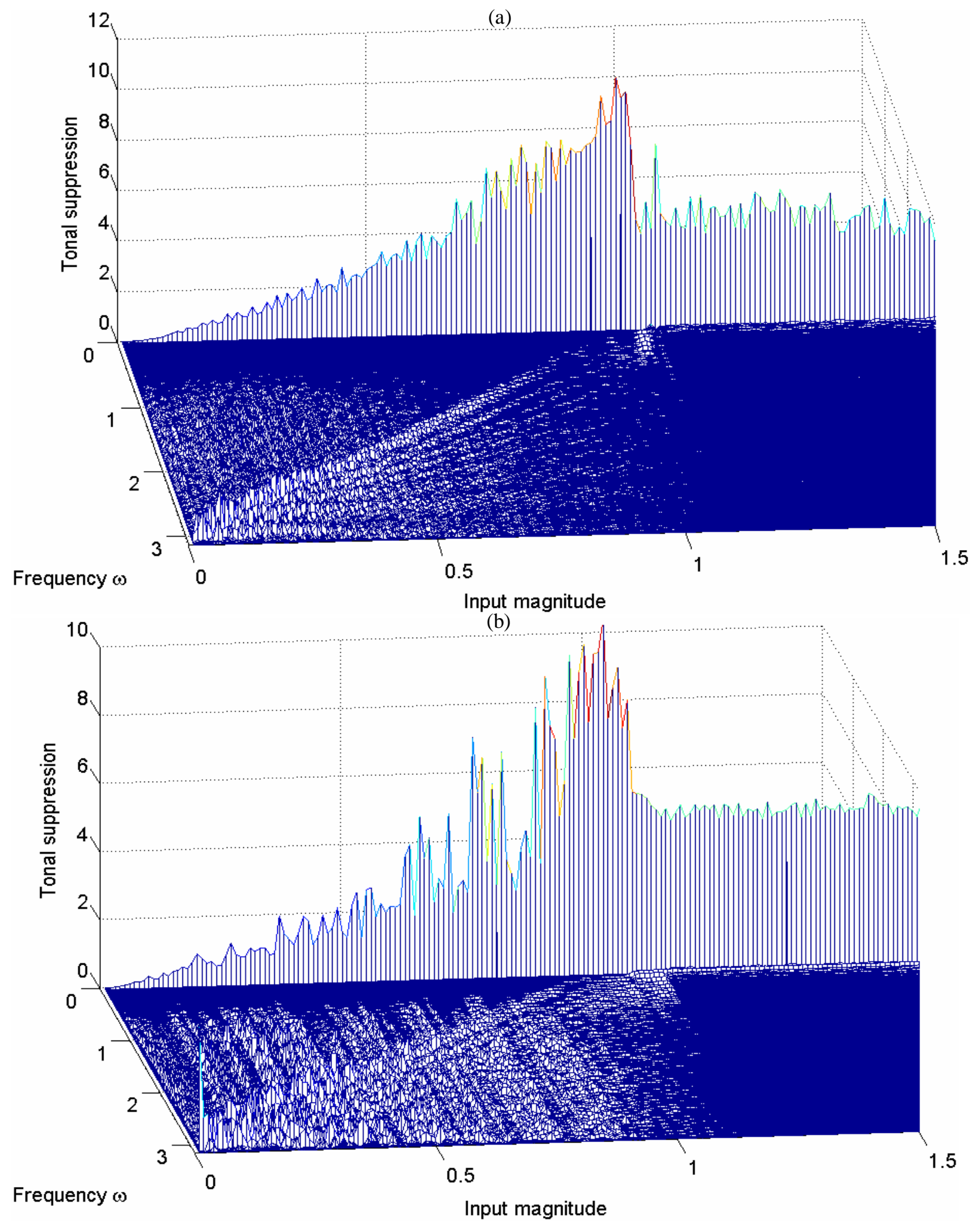


International Journal of Bifurcation and Chaos

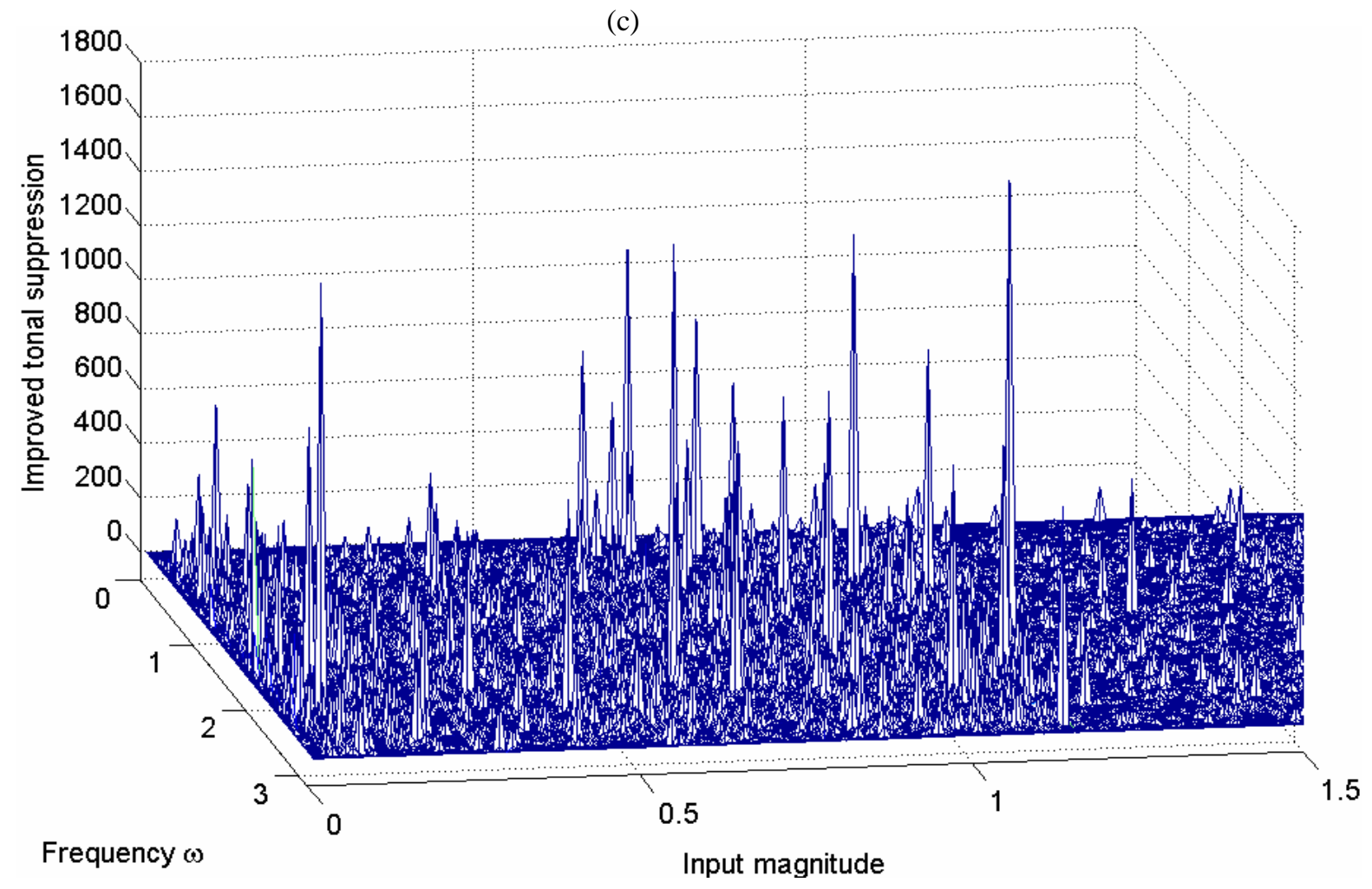

Figure 8. Tonal suppression of (a) the lowpass SDM and (b) the bandpass SDM. (c) Ratio of the improvement of the tonal suppression.

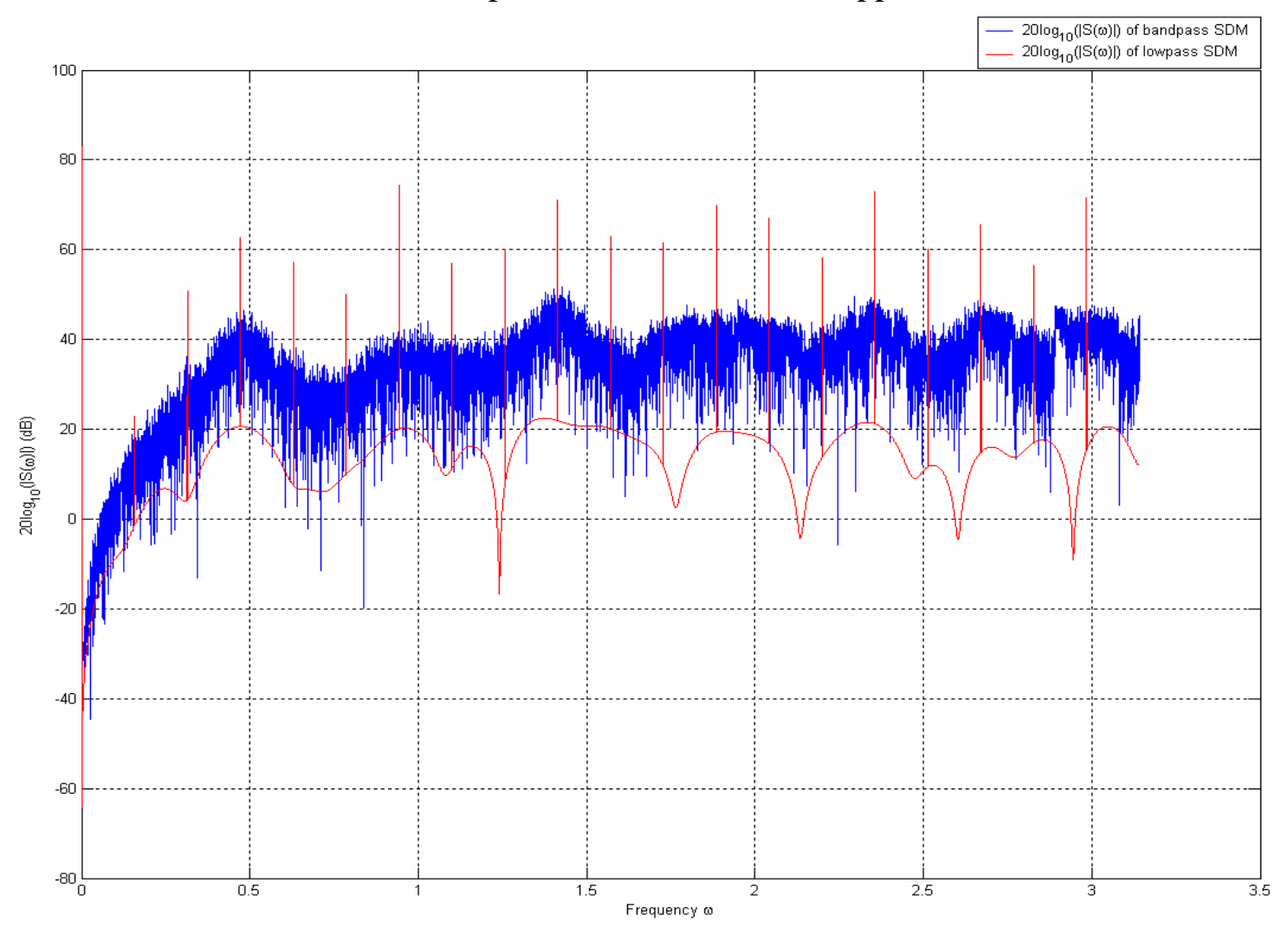

Figure 9. Magnitude spectra of the output sequences of the lowpass and bandpass SDMs. 
International Journal of Bifurcation and Chaos

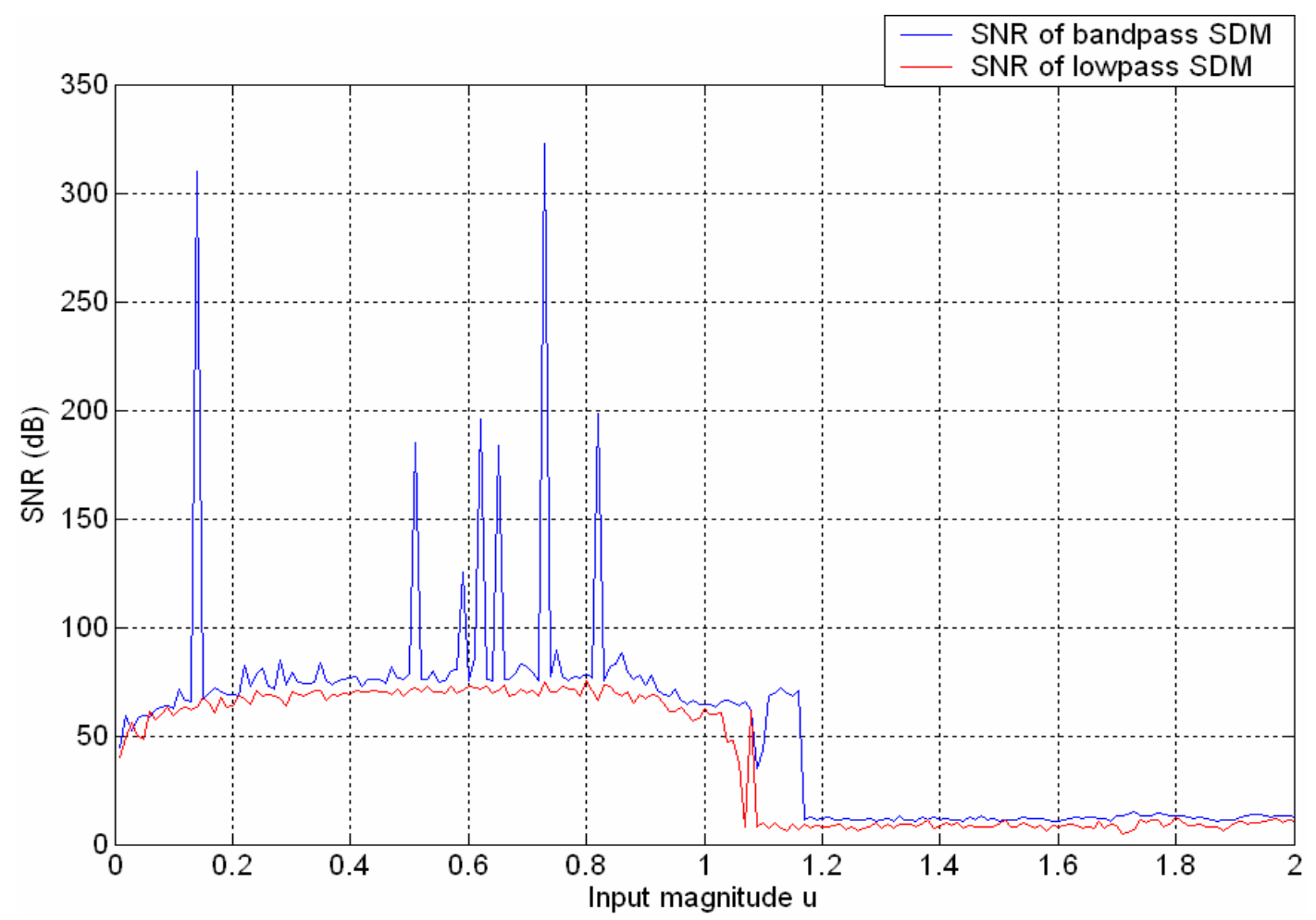

Figure 10. SNRs of the lowpass and bandpass SDMs. 